\title{
Relationship between IL-10 and PD-L1 in esophageal carcinoma tissues and IL-10 down-regulates PD-L1 expression via Met signaling pathway
}

\author{
Qian Qian ${ }^{1}$, Changping Wu ${ }^{1}$, Jianping Chen ${ }^{1}$, Weibing Wang ${ }^{2}$ \\ ${ }^{1}$ Department of Tumor Biological Treatment, The Third Affiliated Hospital of Soochow University, Changzhou 213003, China; ${ }^{2}$ Chinese People's \\ Liberation Army 904 Hospital, Changzhou 214044, China \\ Contributions: (I) Conception and design: Q Qian, C Wu; (II) Administrative support: C Wu, J Chen; (III) Provision of study materials or patients: \\ Q Qian, J Chen; (IV) Collection and assembly of data: Q Qian, W Wang; (V) Data analysis and interpretation: Q Qian, W Wang; (VI) Manuscript \\ writing: All authors; (VII) Final approval of manuscript: All authors. \\ Correspondence to: Changgping Wu. Department of Tumor Biological Treatment, The Third Affiliated Hospital of Soochow University, 185 Juqian \\ Street, Changzhou 213003, China. Email: helen530906@qq.com.
}

Background: Programmed death-ligand 1 (PD-L1) plays a critical role in host immunity in the setting of cancer progression. Interleukin 10 (IL-10) is a multi-cellular, multi-functional cytokine that regulates cell growth and differentiation and participates in inflammatory and immune responses. The purpose of this study was to clarify the relationship between PD-L1 and IL-10 and their clinical importance in esophageal carcinoma (ESCA).

Methods: ESCA patients $(n=100)$ who underwent surgery with preoperative therapy were included in the study. By immuno-histochemical staining, PD-L1, IL-10 and CD8 positive cells were examined in resected specimens. The gene expression levels of PD-L1, IL-10 and Met were detected by qRT-PCR and Western blots, and differentially compared in cancer, adjacent and normal tissues. In cell experiments, the Eca109 and TE-1 cell-lines were incubated with IL-10 or anti-IL-10 antibody, and then PD-L1 and Met expression levels were compared by ELISA and Western blots. The effect of crizotinib and/or IL-10 on the proliferation, invasion and migration of esophageal squamous cell-lines was estimated by CCK8 and transwell assay.

Results: In tumor tissues, the mRNA and protein levels of PD-L1, IL-10 and Met were higher than those in adjacent tissues. The high expression levels of PD-L1 and IL-10 indicated a poor prognosis. IL10 reduced the expression of PD-L1 in esophageal squamous cell-lines via Met signaling. Over-expression of PD-L1 in increased the levels of IL-10, and Met in in ESCA tissue and cell lines. The combination of crizotinib and IL-10 were more effective in inhibiting the proliferation, migration and invasion of esophageal squamous cell lines.

Conclusions: The combination of IL-10 and PD-L1 monoclonal antibody may have therapeutic promise in treating ESCA.

Keywords: Esophageal cell carcinoma; programmed death-ligand 1 (PD-L1); interleukin 10 (IL-10); Met gene; tumor microenvironment

Submitted Oct 13, 2019. Accepted for publication Dec 27, 2019.

doi: 10.21037/jgo.2020.01.06

View this article at: http://dx.doi.org/10.21037/jgo.2020.01.06 


\section{Introduction}

In China, the incidence of esophageal cancer (ESCA) ranks as the fourth most common among cancer-related mortality and is particularly high in provinces of Jiangsu, Henan and Anhui. Worldwide, ESCA is the eighth most common cancer and its incidence rate is ranked sixth among cancerrelated disease with an estimated 456,000 new cases per year $(1,2)$. According to the pathological type, ESCA is mainly divided into esophageal adenocarcinoma (EAC) and esophageal squamous cell carcinoma (ESCC). In China, more than 90 percent of cases are ESCC. Traditional treatments for ESCA are mainly surgery, radiotherapy and chemotherapy $(3,4)$. However, many patients with advanced disease cannot tolerate these treatment methods. Therefore, new treatment strategies are urgently needed for ESCA.

Programmed death-ligand 1 (PD-L1, also called B7$\mathrm{H} 1$ or CD274) is expressed on the surface of many kinds of tumor cells and tumor-infiltrating immune cells (ICs). PDL1 over-expression in cancer cells inhibits the activity of $\mathrm{T}$ lymphocytes $(5,6)$, which results in the blocking of antitumor immunity and immune escape (7). In 2013, the first PD-L1 inhibitor was used in the treatment of lung cancer and achieved amazing clinical results.

In the next few years of drug clinical trials, PD-L1 inhibitors have also achieved good results in the treatment of ESCA (8-10). However, it also has some setbacks during treatment, which predominantly involve side-effects and a low clinical response rate (11-13). Therefore, to improve the clinical efficacy $(14,15)$, the PD-L1 inhibitor is usually combined with chemotherapy $(16,17)$ and/or radiotherapy $(16,18,19)$. Some recent studies have focused on the efficacy of PD-L1 inhibitors combined with immune factors (20-22).

Interleukin 10 (IL-10) is a multifunctional cellular immunosuppressive factor produced by Th2 cells, and plays an important inhibitory role in inflammatory and immunological responses. By inhibiting the activation of natural killer (NK) and T cells, IL-10 enables tumor cells to escape immune surveillance $(23,24)$. However, IL-10 also functions in immune activation, which promotes tumorspecific immune surveillance and reduces the occurrence of pathogenic inflammatory reactions by activating $\mathrm{T}$ and NK cells. Therefore, IL-10 plays a key role in this two-way immune regulatory system (25-27). Some articles indicate that the expression level of PD-L1 is correlated with the expression level of IL-10 in the tumor micro-environment (28-32). However, the relationship between both factors remains unclear.
Mesenchymal-epithelial transition factor (Met) is a tyrosine kinase (TK) receptor for hepatocyte growth factor (HGF) $(33,34)$ (Figure 1A). Met has been reportedly expressed in more than 50 percent of ESCC cases at both the mRNA and protein levels. Activation of the Met gene leads to multiple downstream pathways that promote a tumorigenic phenotype (35).

In our study, we found that in esophageal carcinoma tissue, PD-L1 expression levels were positively correlated with IL-10 expression levels. Over-expression of PD-L1 upregulates IL-10 expression in esophageal carcinoma tissue and cells by positive feedback regulation. IL-10 downregulates the expression levels of PD-L1 in esophageal squamous carcinoma cells-lines by negative feedback regulation. We have also discovered that PD-L1 and IL-10 are linked by the MET signaling pathway (Figure 1B,C).

Thus, our study provides new insights into the functional role of IL-10. The efficacy of combined IL-10 and PD-L1 inhibitor therapy was shown to be better than use of the PD-L1 inhibitor alone.

\section{Methods}

\section{Bioinformatics analysis}

A bioinformatics analysis was conducted by the TCGA database (https://www.cancer.gov/) and the TCGA-based visualization website GEPIA (http://gepia.cancer-pku.cn/). The expression levels of IL-10, PD-L1 and Met genes were compared in ESCA tissues and normal tissues. The expression correlation of PD-L1 and IL-10 genes of ESCA was analyzed. The analyses also included the relationship between IL-10 and overall survival (OS) or disease-free survival (DFS) or between the expression levels of IL-10 and PD-L1 and pathology stage of ESCA.

\section{Clinical samples}

One hundred patients that comprised 60 males and 40 females, with ESCA in the Third Affiliated Hospital of Soochow University from September 2013 to September 2018 were included in the study, which was approved by the Hospital Ethical Review Committee. No patients were treated with any relevant chemotherapy, radiotherapy, targeted therapy, or immunotherapy before surgery. Among 100 ESCA surgical resections, 50 cases were highly differentiated and 50 cases moderately or poorly differentiated. From each patient, three specimens were collected from ESCA tissues, adjacent tissues to the tumor, 
A

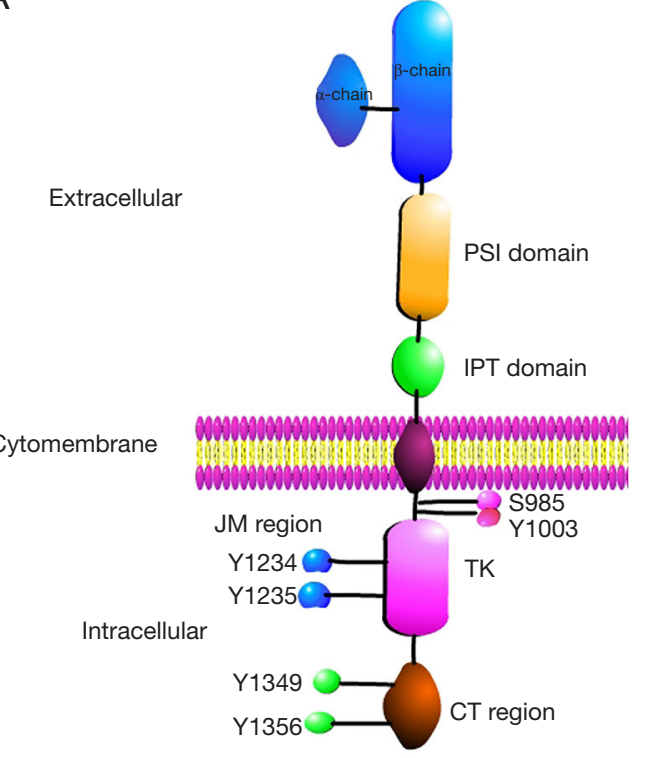

B
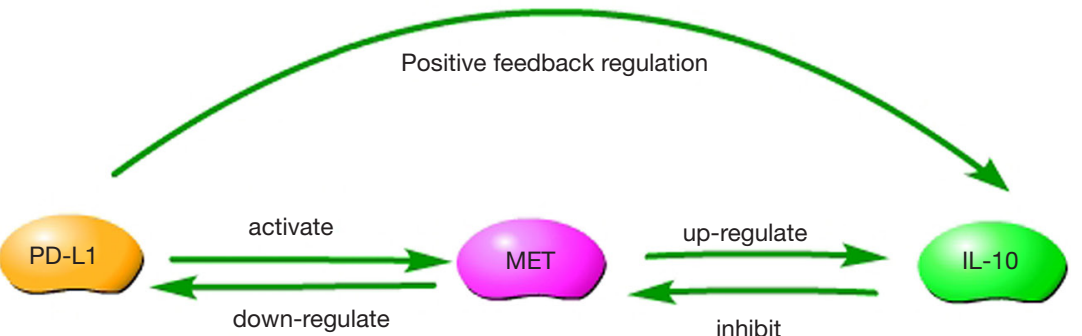

inhibit

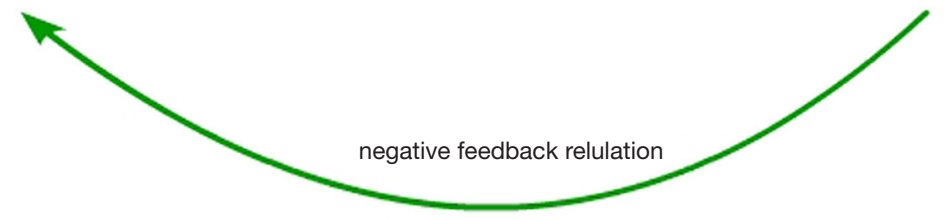

C

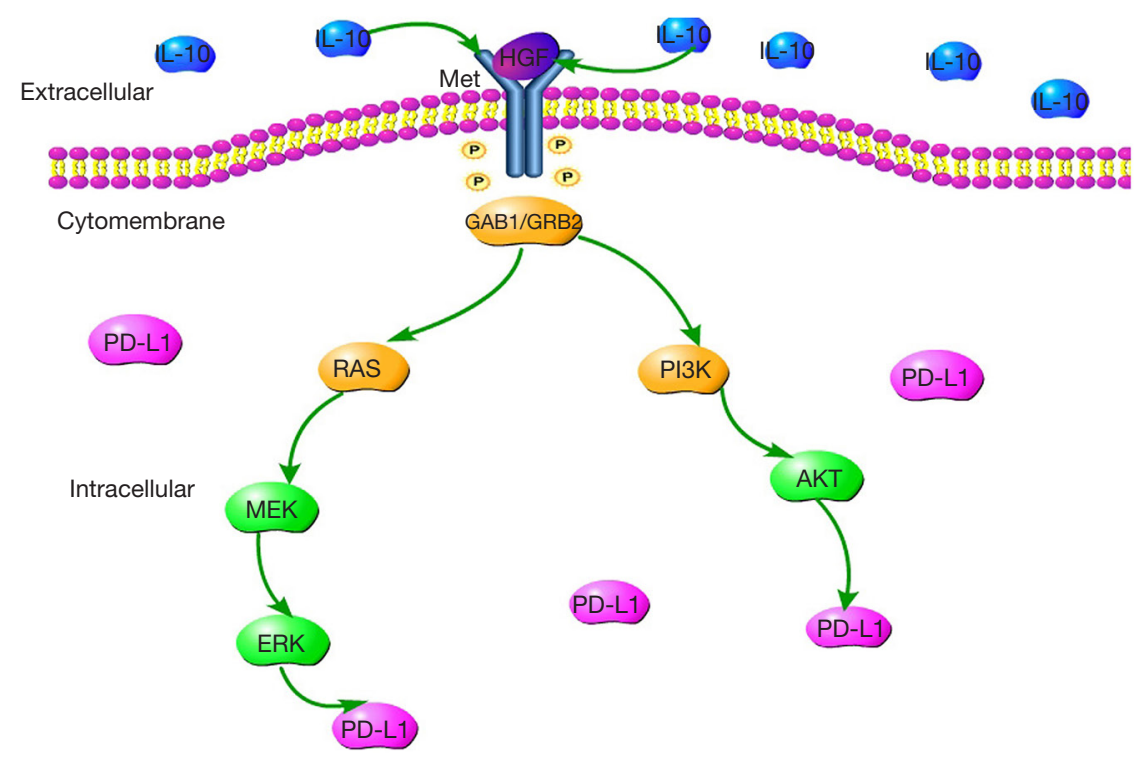

Figure 1 Hand-drawn picture of interactions among PD-L1, Met, and IL-10. (A) Met protein structure diagram: Met is formed by extracellular alpha chain, transmembrane beta chain via disulfide bond heterologous body. The Met receptor includes three functionally distinct domains. That is, the extracellular region, the transmembrane region, and the intracellular region. The intracellular zone includes 3 more functions: intracellular proximal membrane junction domain, a tyrosine kinase catalytic structure with tyrosine kinase activity domain, a C-terminal domain that interacts with a variety of downstream signaling molecules. (B) Dynamic interactions among PD-L1, Met, and IL10. Over-expression of PD-L1 upregulates IL-10 expression in esophageal carcinoma and cells by positive feedback regulation. IL-10 downregulates the expression level of PD-L1 in esophageal carcinoma by negative feedback regulation. (C) IL-10 acts on the HGF (hepatocyte growth factor)/Met signaling pathway, thereby affecting its downstream akt, MAPK signaling pathway, and then down-regulating PD-L1 expression. 
and normal tissues. The specimens were confirmed by pathological diagnosis as ESCA. A portion of the tissues was embedded in paraffin and another part was freshly resected surgical tissues, and stored in liquid nitrogen for further experiments.

\section{Patient follow-up}

Patients were instructed to return for follow-up, including a clinical examination, gastroscopy, thoracic computed tomography, and abdominal ultrasonography, at 3-6 months intervals for one year, and then every 6-12 months thereafter.

\section{Immunochemistry}

The paraffin-embedded tumor samples were sectioned to $5 \mu \mathrm{m}$ slices. The tissue section slides were deparaffinized and rehydrated. For PD-L1, IL-10 and CD8 were stained by corresponding antibodies. Detailed experimental processes can be seen in the supplementary materials. The cell intensity and the rate of positive cells were recorded (Table S1). For data analysis, the final scores of less than eight were defined as low expression and scores of eight or more, were defined as high expression.

\section{Quantitative RT-PCR}

The tissue pieces were first cut into small sections, and then a homogenizer was used to extract the total RNA using the Trizol method. We compared the differences in IL-10, PDL1, and Met mRNA expression among cancerous tissues, adjacent tissues, and normal tissues by quantitative RT-PCR using the described primers (please see Table S2). Detailed experimental processes can be seen in the supplementary materials.

\section{Cell culture}

Human esophageal carcinoma Eca109 and TE-1 celllines were obtained from the Cell Research Center, Third Affiliated Hospital of Soochow University for in vitro studies. Detailed culture conditions can be found in the supplementary materials.

\section{SiRNA construction of esophageal squamous cell-lines}

We used products of obtained from the Gene Pharma Company to build siMET and siPD-L1. Detailed experimental process can be seen in the supplementary materials. SiRNA sequences are listed in Table S3.

\section{Over-expression of PD-L1}

The ESCA cell line over-expressing PD-L1 (lentivirusmediated) is from Cell Research Center, the Third Affiliated Hospital of Soochow University. In the article, it is abbreviated as LV-PD-L1.

\section{ELISA}

One day before drug treatment, cells in logarithmic growth phase were removed and seeded on a six-well plate at a density of $3 \times 10^{4}-10^{5}$ per well. When the cells reach $70 \%$ to $80 \%$ confluency, add different concentrations of IL-10 (Creative BioMart, New York, USA) or anti-IL-10 antibody (Creative Biolabs, New York, USA) to the cells. When the cells were cultured for 24 to 48 hours, the cells were counted by a hemocytometer to ensure that the number of cells per well was approximately the same. PD-L1 Quantikine ELISA kit (R\&D systems, Minneapolis, MN, USA) was used to test the PD-L1 expression level in cell culture supernate or cell lynate of after 24 and $48 \mathrm{~h}$.

At the same time, after successful transfection of siPD-L1 Eca109, siPD-L1 TE-1, and LV PD-L1 Eca109, LV PDL1 TE-1 cell culture supernatant was taken to test the IL10 expression level after 24, 48 hours. Human IL-10 high sensitivity ELISA kit (Multi Sciences, Hangzhou, Zhejiang, China) was used to test the IL-10 expression level.

\section{CCK8}

We used the CCK8 assay (Dojindo, Tokyo, Japan) to detect the proliferation of cells after treatment with Crizotinib (Cell Signaling Technology, Danvers, MA, USA) and Crizotinib combined with IL-10. Lastly, we added Crizotinib or Crizotinib combined with IL-10 to the constructed siMet Eca109 and siMet TE-1. The proliferation of cells was detected by CCK8. Detailed experimental processes can be seen in the supplementary materials

\section{Transwell test}

Analysis of tumor cell migration and invasion was performed by a transwell chamber assay (Corning, New York, USA). About $2-5 \times 10^{3}$ cells, Eca109 or siMet- 
Eca109 cells and TE-1 or siMet-TE-1 cells, were seeded onto the upper chamber in serum-free medium, and then $500 \mu \mathrm{L}$ of medium containing $1 \%$ FBS was added to the lower chamber. Crizotinib or crizotinib combined with IL-10 were added to the lower chamber. After 24 or $48 \mathrm{~h}$ incubation, the cells were fixed by incubating in $4 \%$ formaldehyde at room temperature for $5 \mathrm{~min}$. After staining with crystal violet, the number of cells that migrated or invaded were observed under the light microscope.

\section{Western blots}

PD-L1 expression levels, MET signaling pathway-related molecules (MET and phospho-MET) and their downstream MAPK signaling pathway-related molecules (akt, phosphoakt, Mek, phospho-Mek, Erk, phospho-Erk) were analyzed by Western blotting. Meanwhile, we performed Western immunoblotting on ESCA cells that over-expressed or displayed knocked-down PD-L1 gene expression to detect differences in IL-10 expression levels.

After that, crizotinib or crizotinib combined with IL10 were added to the cells. Met signaling pathway-related molecules (Met and phospho-Met) were analyzed. In our tissue experiments, the tissue pieces were first cut into small sections, and then a homogenizer was used to extract tissue protein using the protein extraction agent. We compared the differences in IL-10, PD-L1, and Met expression among cancerous tissues, adjacent tissues, and normal tissues. Detailed experimental processes can be seen in the supplementary materials.

\section{Statistical methodology}

Quantitative data were presented as mean \pm SD . Comparison of the differences between the two groups of data used the $\chi^{2}$ test. Kaplan-Meier curves were generated for OS and DFS. SPSS version 25.0 statistical software was used to assess Cox regression analyses. Alpha values of $\mathrm{P}<0.05$ were considered statistically significant. All data was analyzed by SPSS version 25.0 or Graphpad 5.0 software. Western blotting gray values were analyzed by Image $\mathbf{J}$ software.

\section{Results}

\section{Bioinformatics analysis results}

The gene expression levels of PD-L1, IL-10 and Met in ESCA tissues tend to higher than those in normal tissues.
There was no statistically significant difference for either PD-L1 or IL-10 $(\mathrm{P} \geq 0.01)$ (Figure $2 A, B)$, although a statistical difference was noted for Met $(\mathrm{P}<0.01)$ (Figure $2 C$ ). There was a positive correlation between PD-L1 (CD274) and IL-10 expression (Figure 2D), and the expression level of the IL-10 gene did not affect OS or DFS of patients with ESCA (Figure 2E,F). IL-10 gene expression was associated with the pathological stage of ESCA $(\mathrm{P}=0.0328$; Figure $2 G)$. $\mathrm{PD}-\mathrm{L} 1$ expression was not associated with the pathological stage of ESCA $(\mathrm{P}=0.423$; Figure $2 H)$.

\section{Patient characteristics and immunobistochemistry}

The demographic and clinicopathologic characteristics of 100 ESCA patients are summarized in Tables 1 and 2. By the immunohistochemistry (IHC) evaluation, PD-L1 was mainly expressed in the membrane and cytoplasm (Figure $3 A, B$ ) and IL-10 in the cytoplasm of cancer cells (Figure $3 C, D$ ). CD8 was mainly located on the surface of the T lymphocyte membrane (Figure $3 E, F$ ). Among the 100 ESCA cases, 72 cases $(72.0 \%)$ exhibited high PD-L1 expression levels and 69 cases $(69.0 \%)$ displayed high IL-10 levels; however, only 15 cases exhibited high expression levels of CD8+ T lymphocyte in tumor tissues. Conversely, 52 cases displayed high expression levels of CD8+ T lymphocyte in adjacent tissues. A comparison of the clinicopathological characteristics according to the PD-L1 expression levels is listed in Table 1. Higher PD-L1 expression levels tended to be related to a patients of a higher age group $(\mathrm{P}=0.000)$, poor tumor differentiation $(\mathrm{P}=0.014)$, advanced $\mathrm{T}$ stage $(\mathrm{P}=0.000)$, pathology stage $(\mathrm{P}=0.001)$ higher IL-10 expression levels $(\mathrm{P}=0.038)$ and higher CD8 expression levels $(\mathrm{P}=0.008)$. Higher IL-10 expression levels were associated with poor tumor differentiation $(\mathrm{P}=0.03)$, advanced $\mathrm{T}$ stage $(\mathrm{P}=0.001), \mathrm{N}$ pathological stage $(\mathrm{P}=0.02)$, the pathologic stage $(\mathrm{P}=0.000)$, and higher $\mathrm{PD}-\mathrm{L} 1$ expression $(\mathrm{P}=0.000)$ of ESCA (Table 2).

\section{Relationship between the IL-10 expression level and survival periods}

The high IL-10 expression level group tended to have shorter survival periods than those with low IL-10 expression levels. The 5 -year DFS $(\mathrm{P}=0.165)$ and $\mathrm{OS}$ rate $(\mathrm{P}=0.18)$ in ESCA patients with the IL-10 high expression level was poor as compared those with low expression levels, but without statistical significance (Figure $3 G, H$ ). 
A

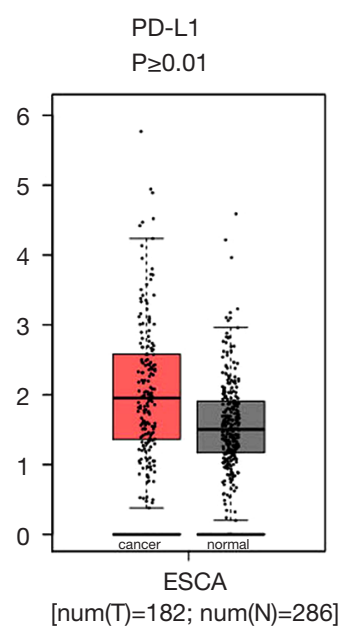

D

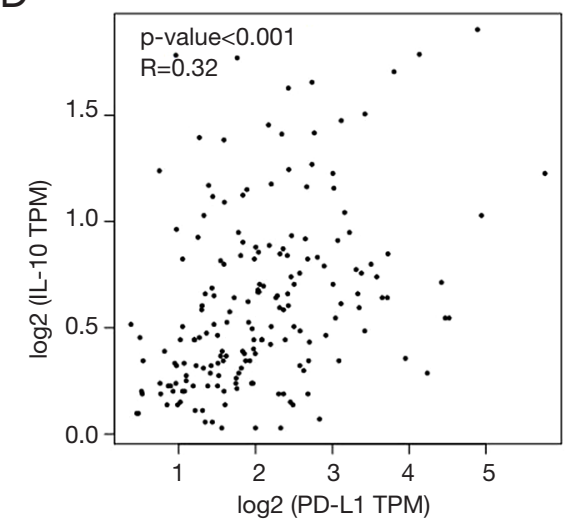

B

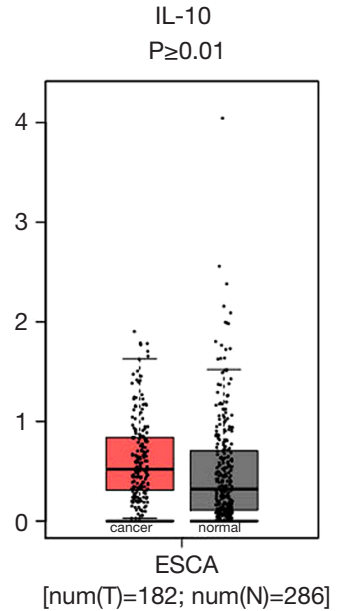

E

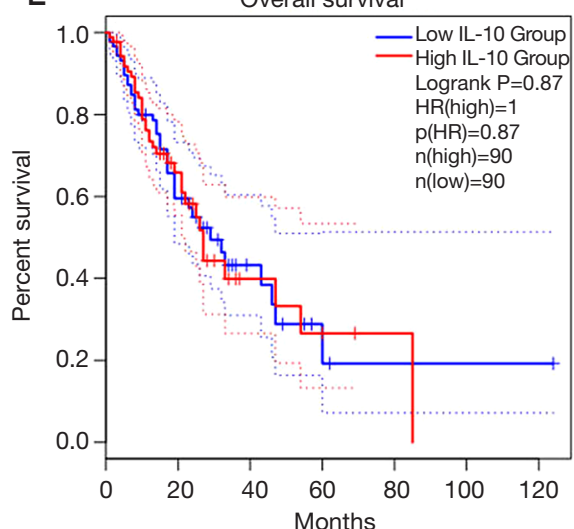

C

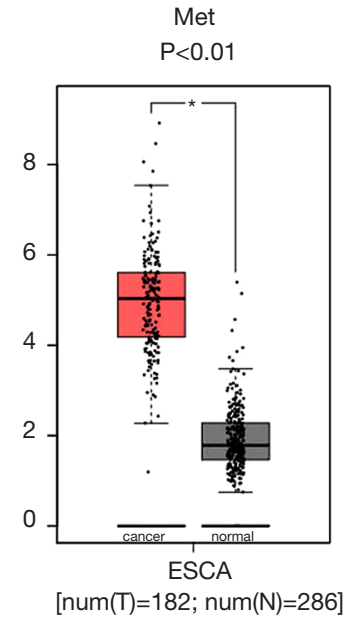

$\mathrm{F} \quad$ Disease free survival

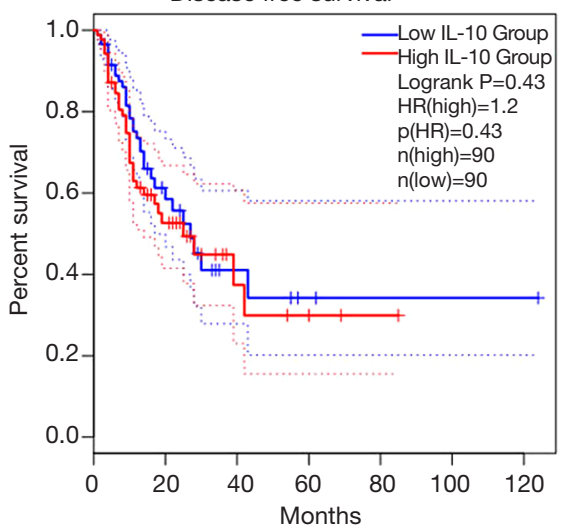

G

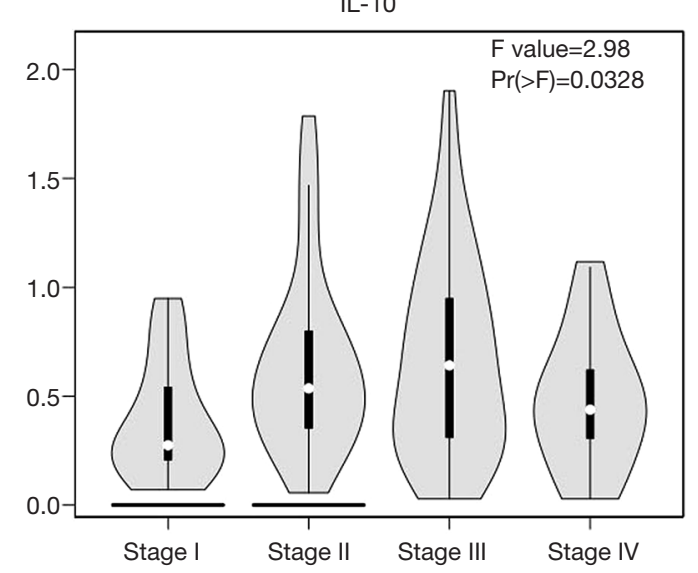

$\mathrm{H}$

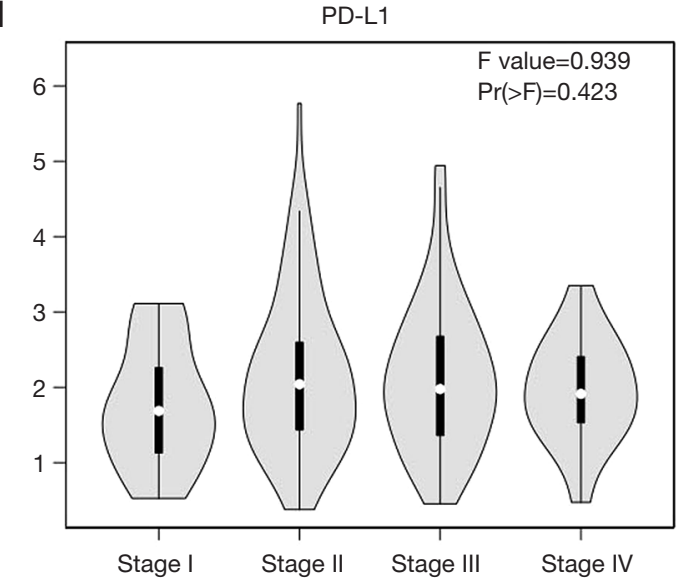

Figure 2 Bioinformatics results analyzed by TCGA database. (A,B,C) Comparison of the gene expression levels of programmed death-ligand 1 (PD-L1), interleukin 10 (IL-10) and Met in esophageal cancer tissues and in normal tissues by TCGA related database. (D) Correlation analysis of PD-L1 and IL-10 gene expression in esophageal carcinoma. (E,F) Overall survival (OS) and disease-free survival (DFS) according to IL-10 expression of patients with esophageal cancer. (G,H) Relationship between IL-10 and PD-L1 gene expression and pathological stage of esophageal cancer. *, $\mathrm{P}<0.01$ compared with normal tissue. 
Table 1 Demographic and clinical characteristics of PD-L1 expression in patients with ESCA

\begin{tabular}{|c|c|c|c|c|c|}
\hline Characteristic & $\mathrm{n}$ & \multicolumn{2}{|c|}{ PD-L1 } & $\chi^{2}$ & $\mathrm{P}$ \\
\hline Sex & & & & 2.116 & 0.146 \\
\hline Male & 60 & 20 & 40 & & \\
\hline Female & 40 & 8 & 32 & & \\
\hline$<60$ & 24 & 5 & 19 & & \\
\hline$\geq 60$ & 76 & 23 & 53 & & \\
\hline Tumor location & & & & 3.191 & 0.203 \\
\hline Upper esophagus & 15 & 5 & 10 & & \\
\hline High & 50 & 20 & 30 & & \\
\hline Moderate/low & 50 & 8 & 42 & & \\
\hline T stage & & & & 38.762 & 0.000 \\
\hline $\mathrm{T} 1 / \mathrm{T} 2$ & 32 & 22 & 10 & & \\
\hline T3/4 & 68 & 6 & 62 & & \\
\hline $\mathrm{N}$ stage & & & & 0.588 & 0.443 \\
\hline No & 54 & 20 & 34 & & \\
\hline High expression & 69 & 7 & 62 & & \\
\hline \multicolumn{6}{|l|}{ CD8 } \\
\hline Low expression & 30 & 3 & 27 & 6.888 & 0.008 \\
\hline High expression & 70 & 25 & 45 & & \\
\hline
\end{tabular}

$\mathrm{P}<0.05$ was considered the difference has statistical significance.

The 5-year DFS $(\mathrm{P}=0.048)$ and OS $(\mathrm{P}=0.046)$ in ESCA patients with low IL-10/PD-L1 expression levels were longer than those with high IL-10/PD-L1 expression levels (Figure 31,7) Furthermore, we performed a multi-factor Cox survival analysis on IL-10/PD-L1, T stage, and N stage, and showed that the combination of IL-10 and PD-L1 was an independent prognostic factor, just as the $\mathrm{T}$ stage and $\mathrm{N}$ stage are equally known (Table 3).

Positive correlation between IL-10 and PD-L1 expression levels in tissues

Among the 100 ESCA cases, the high expression level of PD-L1 was observed in 72 tumor tissues $(72.0 \%)$ 
Table 2 Demographic and clinical characteristic of IL-10 expression in patients with ESCA

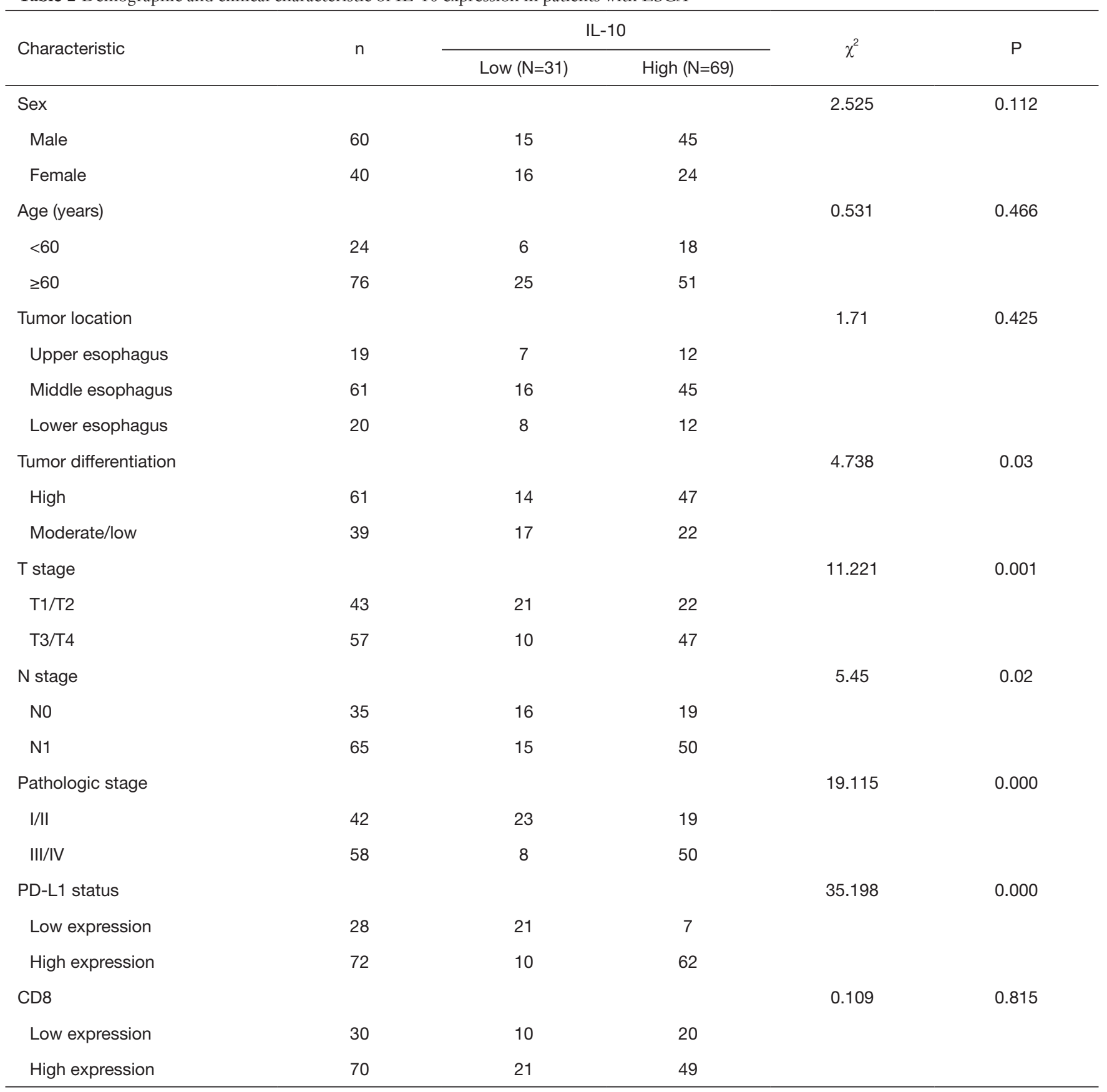

$\mathrm{P}<0.05$ was considered the difference has statistical significance.

and in 25 adjacent tissues (25.0\%). In tumor tissues, the expression of $\mathrm{PD}-\mathrm{L} 1$ was significantly higher than that of adjacent tissues $(\mathrm{P}=0.000$; Table 4). Meanwhile, the high expression levels of IL-10 were observed in 69 tumor tissues $(69.0 \%)$ and in 28 adjacent tissues (28.0\%). In tumor tissues, the expression of IL-10 was significantly higher than that of adjacent tissues $(\mathrm{P}=0.000$; Table 5). By Pearson correlation analysis, there was a positive correlation between the expression levels of PD-L1 and IL-10 in tumor and adjacent tissues (Figure $4 A, B$ ), and a negative correlation was found between the expression levels of PD-L1and CD8 in 
PD-L1
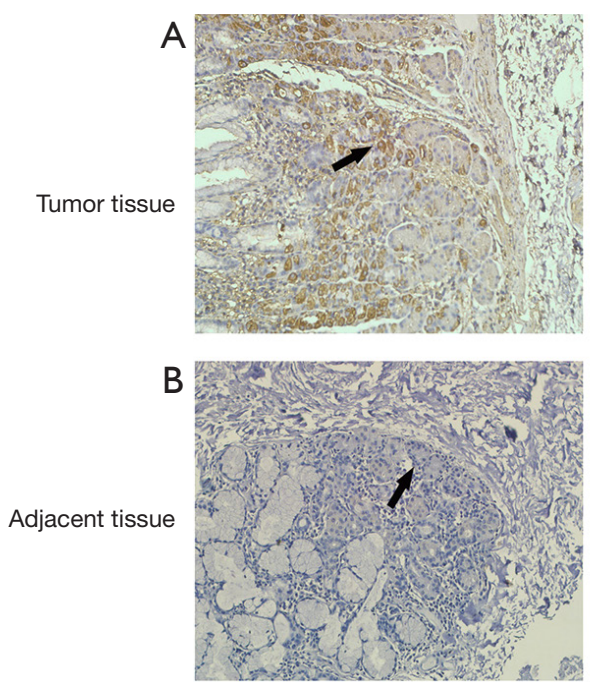

G
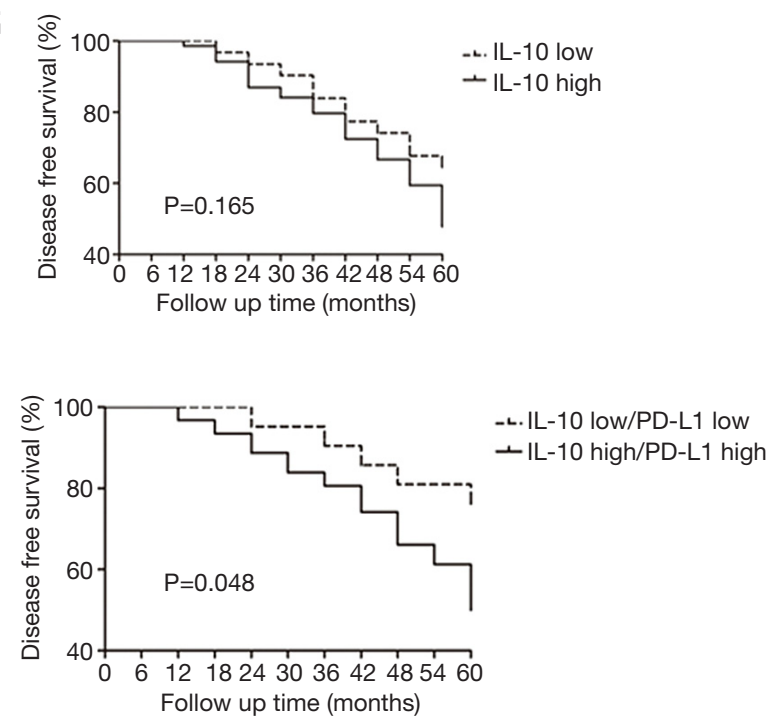

IL-10
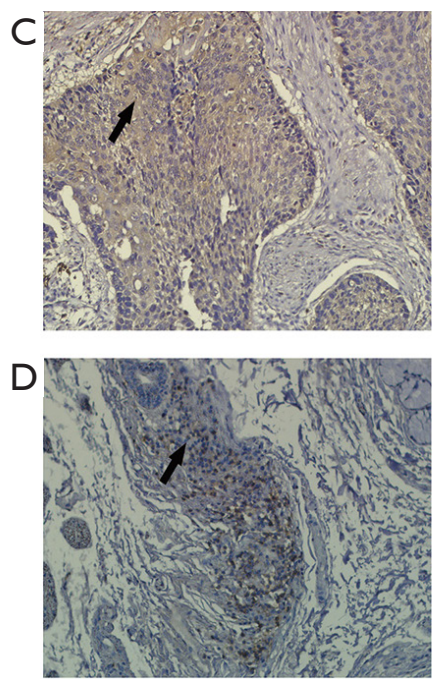

CD8

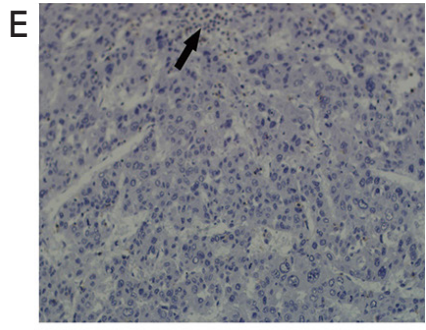

$\mathrm{F}$

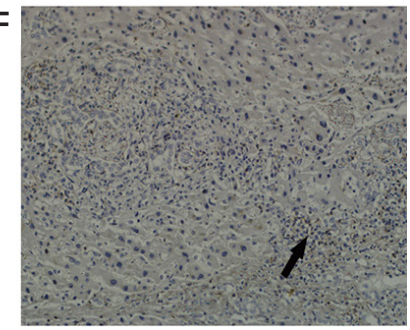

$\mathrm{H}$

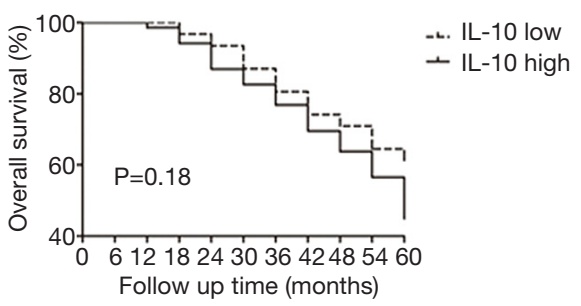

J

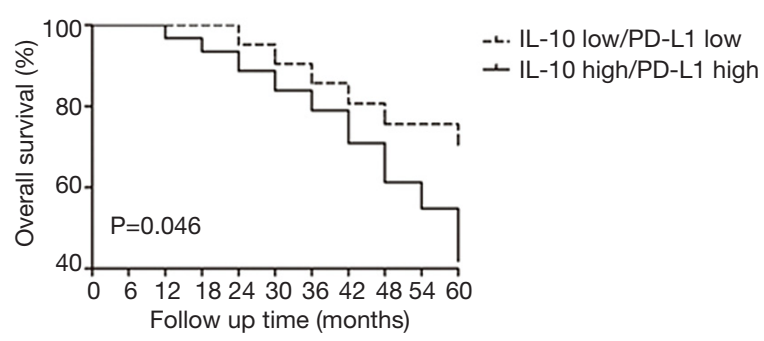

Figure 3 Expression of PD-L1, IL-10 and CD8 and prognosis according to the PD-L1 and IL-10 expression levels in patients with ESCA. $(A, B)$ Representative images of immunohistochemical staining with anti-PD-L1 in esophageal cancer tissues (A) and adjacent tissues (B). (C,D) Representative images of anti-IL-10 in esophageal cancer tissues (C) and adjacent tissues (D). (E,F) Representative images of anti-CD8 in esophageal cancer tissues (E) and adjacent tissues (F). The arrows are for positive regions. Magnification: ×200. (G,H) Overall survival (OS) and disease-free survival (DFS) of patients with esophageal squamous cell carcinoma in relation to IL-10 expression status. (I,J) OS and DFS rates of patients in relation to the PD-L1 and IL-10 expression status.

tumor and adjacent tissues (Figure 4C,D). Moreover, when analyzing both mRNA by PCR and protein expression by Western blots, we found expression levels of PD-L1 and IL-10 in tumor tissues were higher than those found in adjacent and normal tissues, and the expression levels of Met in the tumor tissues were higher than those found in adjacent tissues (Figure 4E,F,G,H).

\section{Correlation between IL-10 and PD-L1 expression levels in ESCA cell-lines}

ELISA studies showed that in both Eca109 and TE-1 cells, the over-expression of PD-L1 expressing cells increased the IL-10 expression levels. The IL-10 expression levels in siPD-L1 Eca109 and TE-1 cells was lower than that 
Table 3 COX multifactor survival analysis

\begin{tabular}{lcccccc}
\hline & $\beta$ & SE & $\mathrm{P}$ & \multicolumn{2}{c}{$95 \% \mathrm{Cl}$} & \multicolumn{2}{c}{ Lower } & & \multicolumn{2}{c}{ Upper } \\
\cline { 5 - 7 } Group & 1.258 & 0.629 & 0.046 & 3.517 & 1.024 & 12.074 \\
$\mathrm{~T}$ & -1.891 & 0.794 & 0.017 & 0.151 & 0.032 & 0.715 \\
$\mathrm{~N}$ & 3.211 & 0.92 & 0 & 24.793 & 4.083 & 150.561 \\
\hline
\end{tabular}

Group: PD-L1 low/IL-10 low vs. PD-L1 high/IL-10 high; T: T stage of TMN; N: N stage of TMN. P<0.05 was considered the difference has statistical significance.

Table 4 Comparison of PD-L1 expression levels in patients with ESCA

\begin{tabular}{lcccc}
\hline Tissue & $\mathrm{n}$ & PD-L1 high expression & PD-L1 low expression & $\chi^{2}$ \\
\hline Esophageal cancer tissue & 100 & 72 & 28 & 44.22 \\
Adjacent tissue & 100 & 25 & 75 & 0.000 \\
\hline
\end{tabular}

$\mathrm{P}<0.05$ was considered the difference has statistical significance.

Table 5 Comparison of IL-10 expression levels in patients with ESCA

\begin{tabular}{lccccc}
\hline Tissue & $\mathrm{n}$ & $\mathrm{IL}-10$ high expression & IL 10 low expression & $\chi^{2}$ & $\mathrm{P}$ \\
\hline Esophageal cancer tissue & 100 & 69 & 31 & 33.65 \\
Adjacent tissue & 100 & 28 & 72 & 0.000 \\
\hline
\end{tabular}

$\mathrm{P}<0.05$ was considered the difference has statistical significance.

found in the LV-PD-L1 and control group (Figure $5 A, B)$. The IL-10 expression level was time-dependent. Detection by Western blots showed that the knockdown of PDL1 (siPD-L1) decreased IL-10 levels, and yet the overexpression of PD-L1 (LV-PD-L1) increased the levels and appearance of IL-10, Met and phospho-Met cells, when compared with control cells (Figure 5C,D) .

Detecting by ELISA, PD-L1 was expressed in both cell culture supernatant and cell lysates of Eca109 and TE-1 cells. The PD-L1 expression level was significantly higher in cell lysates than that found in the supernatant (Figure $6 A, B, C, D)$. At the protein level, IL-10 inhibited PDL1 expression in both cell-lines, and anti-IL-10 antibody promoted the expression of PD-L1 in both cell-lines (Figure $6 A, B, C, D, E, F)$. IL-10 acted by inhibiting the Met signaling pathway and the downstream MAPK signaling pathway (Figure 6E,F). Knockdown of Met gene expression in both cell-lines had no significant effect on PD-L1 expression levels after different treatments (Figure 6G). The above phenomena were concentration and timedependent.

\section{Effect of IL-10 and crizotinib on proliferation, migration} and invasion of ESCA cells

When compared with crizotinib treatment alone, the combination of IL-10 and crizotinib more potently inhibited migration and invasion of ESCA cells (Figure $7 A, B$ ). After knockdown of the Met gene, there was no significant difference found in terms of the migration and invasion of ESCA cells when comparing any of the treatments with combination therapy of both drugs and crizotinib alone (Figure 7C,D). Moreover, Western blot assay showed that the combination of crizotinib and IL-10 was more potent at inhibiting PD- L1 expression than use of crizotinib alone. This effect worked via the Met signaling pathway (Figure $7 E, F$ ). Similarly,the combination of IL-10 and crizotinib more potently inhibited proliferation of ESCA cells (Figure 8A,B). After knockdown of the Met gene, there was no significant difference found in terms of the proliferation of ESCA cells (Figure 8C,D).

\section{Discussion}

Bioinformatics is a new subject and plays an important 
A

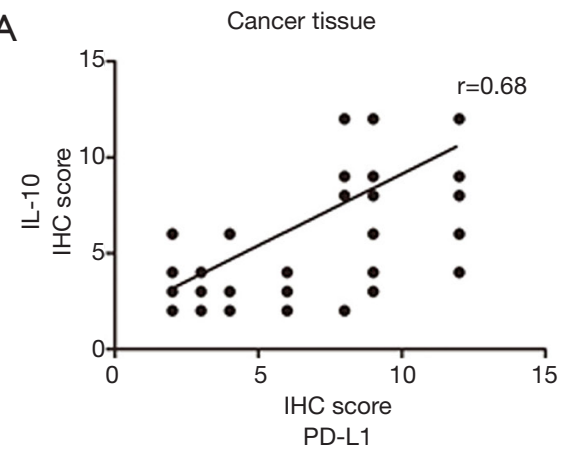

C

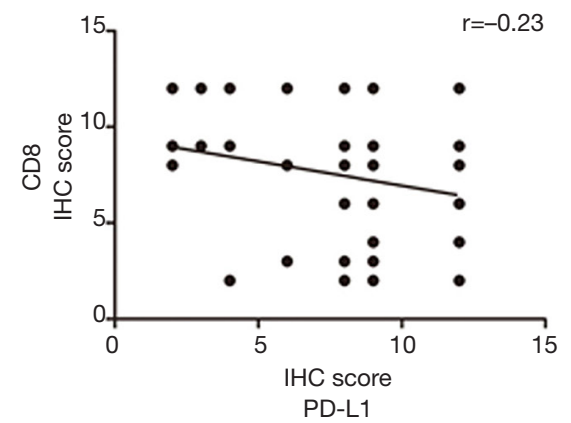

$\mathrm{E}$

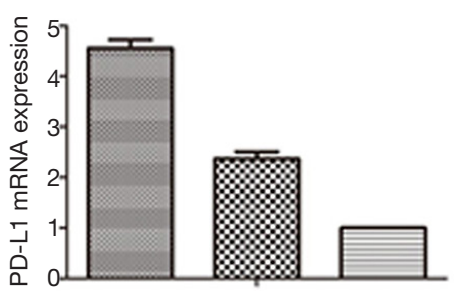

G

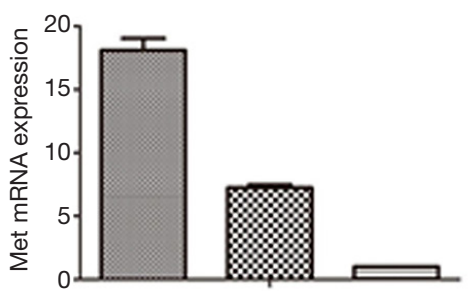

B Adjacent tissue

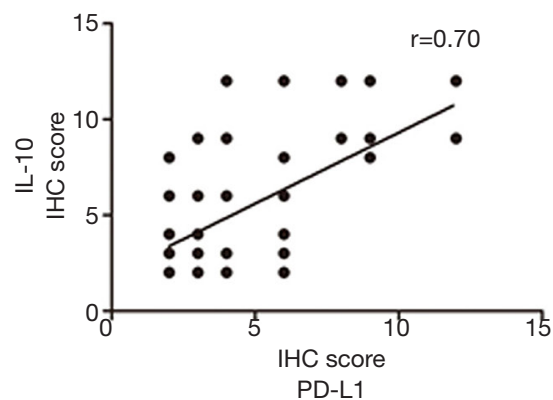

D Adjacent tissue

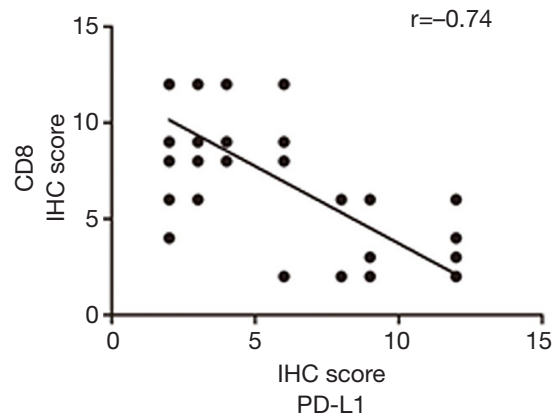

$\mathrm{F}$

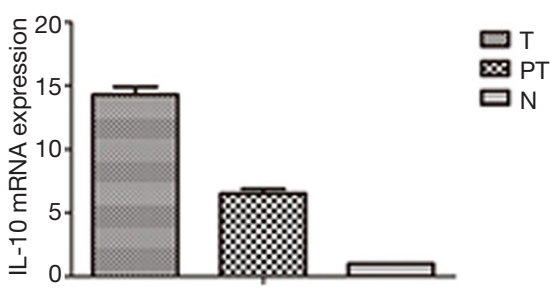

$\mathrm{H}$

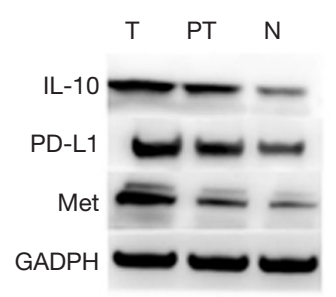

Figure 4 Relationship between the expression levels of PD-L1 IL-10 and CD8, and comparison of PD-L1, IL-10 and Met in ESCA. (A,B) Pearson correlation analysis of the expression levels of PD-L1 and IL-10 in esophageal cancer tissues (A) and adjacent tissues (B). (C,D) Pearson correlation analysis of expression level of PD-L1 and CD8 in esophageal cancer tissues (C) and adjacent tissues (D). (E,F,G,H) Comparison of IL-10, PD-L1 and Met mRNA expression levels in esophageal cancer tissues, adjacent tissues and normal tissues by quantitative real-time PCR and Western blots. $\mathrm{P} \leq 0.05$ was considered statistically significant. T: esophageal cancer tissues; PT: adjacent tissues; N: normal tissues. 
A

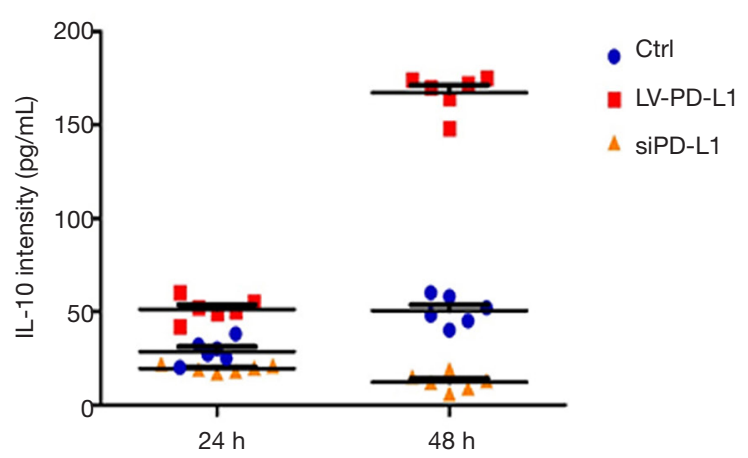

B

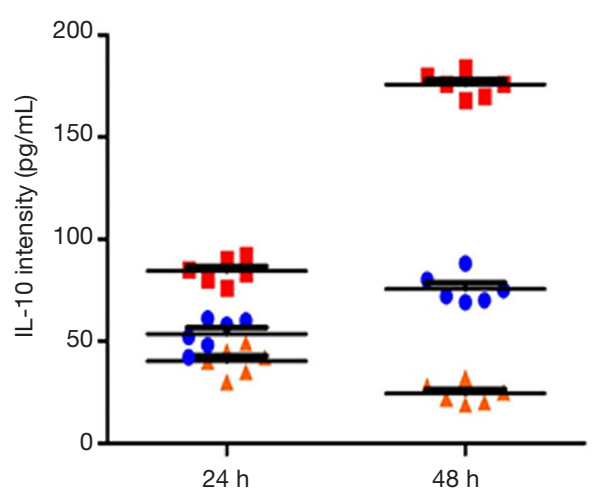

C Eca109 cells
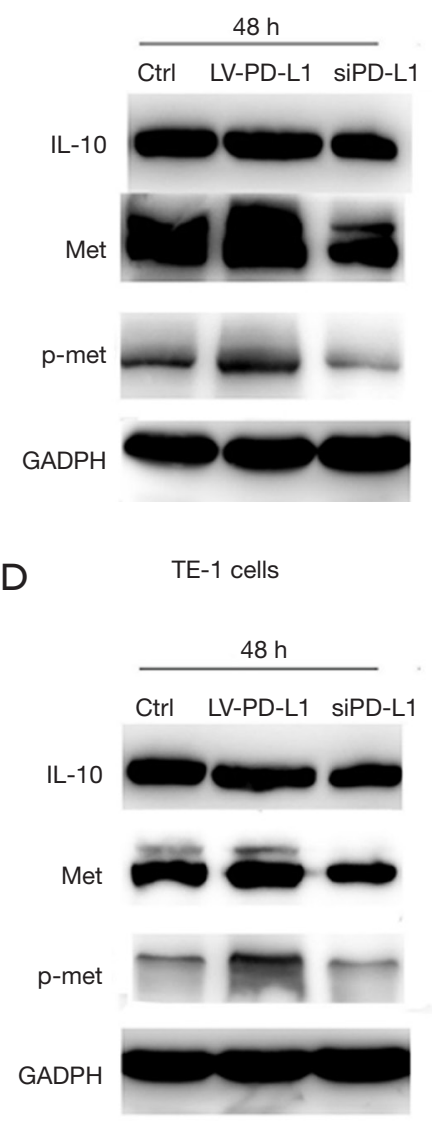

Figure 5 Effects of PD-L1 knockdown and overexpression expression on the expression levels of IL-10, Met and phosphor-Met in esophageal cancer cell lines. Eca109 (A,C) and TE-1 cells (B,D) were transfected with PD-L1 siRNA to knock down the expression of PD-L1 (siPD-L1), and with lentivirus to over-express PD-L1 (LV-PD-L1). Cells without any transfection were as the control. The IL-10 levels were detected by ELISA (A,B). The levels of IL-10, Met, p-Met were measured by Western blots. GADPH was used as the loading control (C,D).

role in current cancer research for studying the collection, processing, storage, dissemination, analysis and interpretation of biological information. TCGA is a cancer gene database developed by the National Cancer Institute and the National Human Genome Research Institute in the United States. Using the relevant database, we have found a correlation between the expression levels of PD-L1 and IL-10 in patients with esophageal carcinoma (ESCA), which provides novel ideas and a basis for our experimental research.

We also discovered that higher IL-10 expression levels are associated with poor tumor differentiation, advanced $\mathrm{T}$ stage, $\mathrm{N}$ stage pathological stage, advanced pathologic stage, and higher PD-L1 expression of ESCA. ESCA patients with high IL-10 expression levels usually had a shorter OS and DFS, but with low IL-10 expression levels had longer OS and DFS. However IL-10 is not an independent prognostic factor in patients with ESCA. The results did not match the results of a previous study by Yang's study. (36).

Our study showed that IL-10 cannot be an independent factor. On the other hand, higher PD-L1 expression levels are associated with higher age, poor tumor differentiation, advanced T stage, pathology stage, higher IL-10 expression levels and higher CD8 expression levels. In addition, PD-L1 combined with IL-10 might be an independent prognostic factor in patients with ESCA. Patients in the low IL-10/ low PD-L1 group had an improved prognosis than those in the high IL-10/high PD-L1 group. Results were consistent 
A Eca109 cell lysates

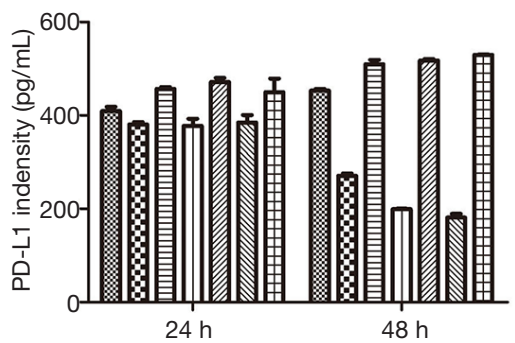

C

TE-1 cell lysates

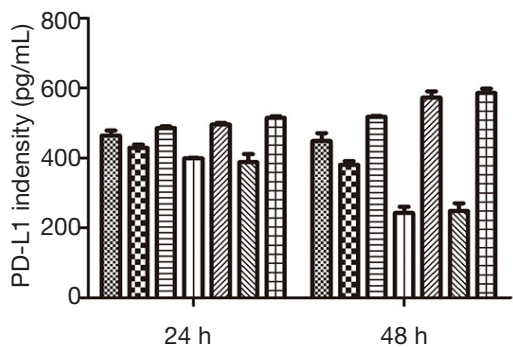

$E$ Eca109 cell lysates $48 \mathrm{~h}$ $0.5 \stackrel{0.7}{0} \quad \underline{1.0}$

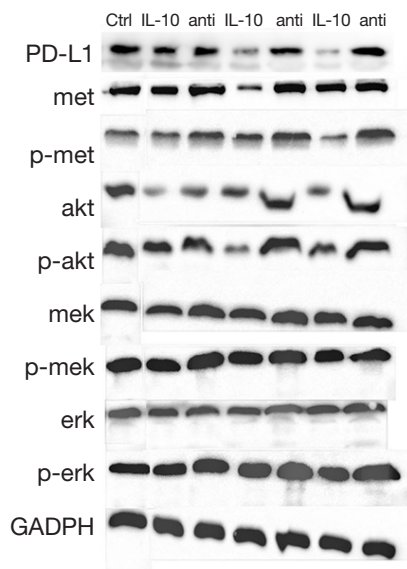

Ctrl

IL-10(0.5)

D anti-IL-10(0.5)

mIL-10(0.7)

anti-IL-10(0.7)

IL-10(1.0)

manti-IL-10(1.0)
B

Eca109 cell supernatant

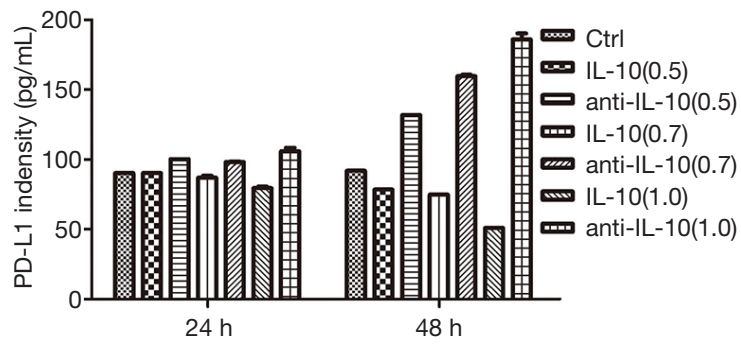

D

TE-1 cell supernatant
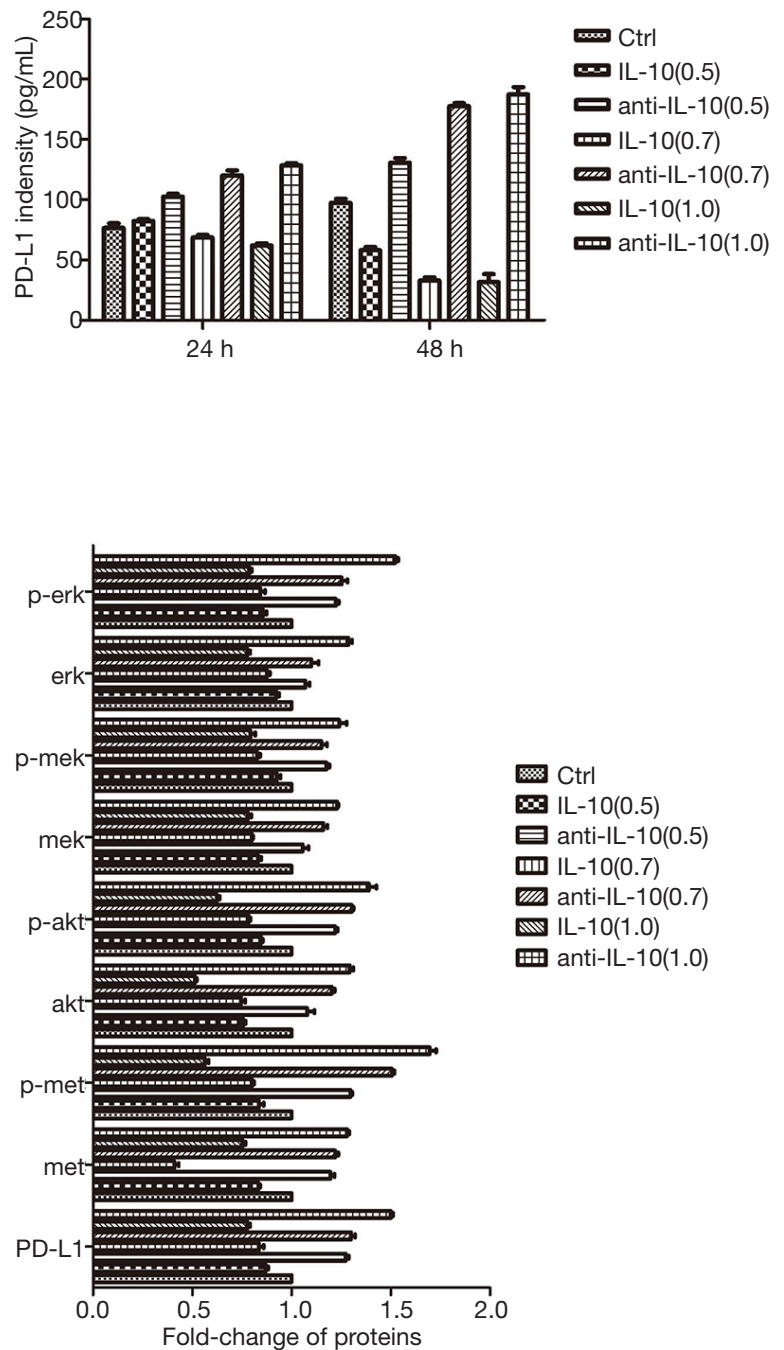
$\mathrm{F}$

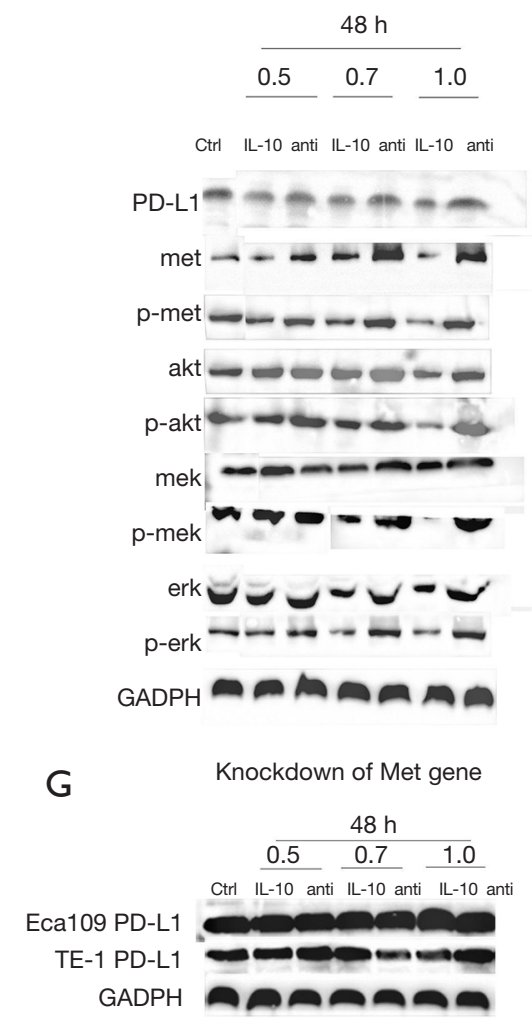

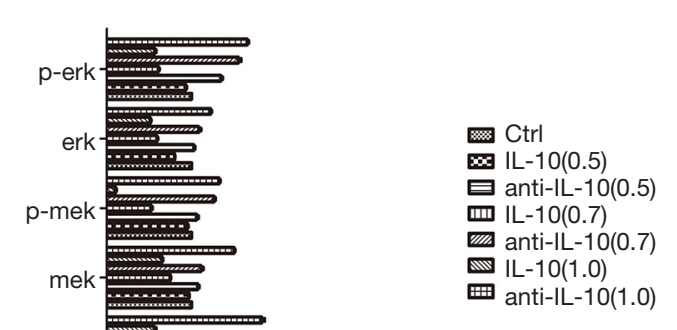
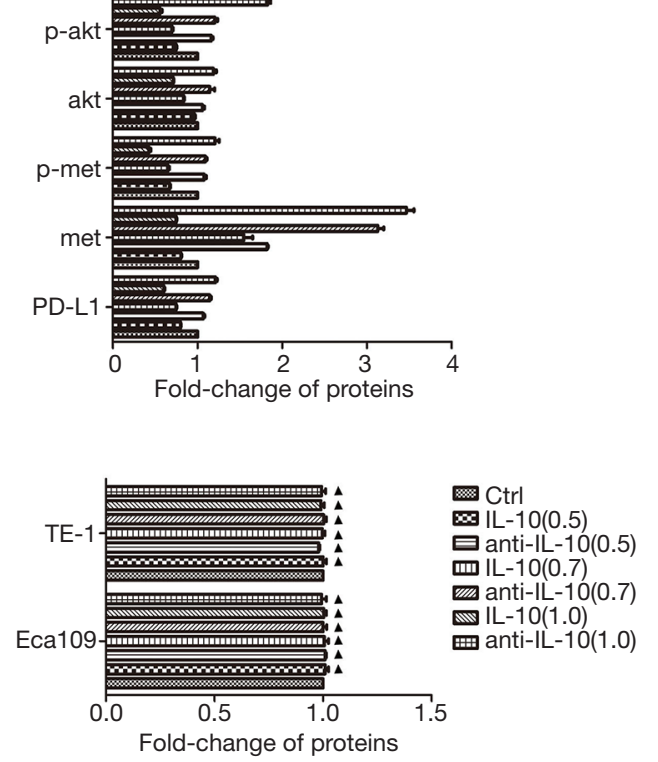

Figure 6 Effects of IL-10 or anti-IL-10 on PD-L1 expression in esophageal cancer cell lines. (A,B,C,D) ELISA experiments for the effects of different concentrations (from $0.5 \mathrm{uL} /$ plate to $1.0 \mathrm{uL} / \mathrm{plated}$ ) of IL-10 or anti-IL-10 on the expression of PD-L1 in cell culture supernatant or cell lysates of Eca109 and TE-1 cell lines. (E,F) Western blots for the effects of different concentrations of IL-10 or antiIL-10 on PD-L1, Met signal pathway and downstream MAPK signaling pathway in Eca109 and TE-1 cell lines. (G) Western blots for the effects of different concentrations of IL-10 or anti-IL-10 on PD-L1 after Met gene was knocked down. $\mathbf{\Lambda}, \mathrm{P} \geq 0.05$ compared with crizotinib group. The remaining data differences are statistically significant.

with prior studies (37-39).

At the transcriptional and translational levels, the higher expression of IL-10 and PD-L1 were observed in cancer tissues, when compared with those in normal tissues, which is consistent with previous studies (40-42). In tumor and adjacent tissues, the PD-L1 expression level was positively correlated with IL-10 expression levels, and was negatively correlated with the CD8+ T cells. Moreover, in cancer tissues, the expression levels of Met at both the transcriptional and translational levels was significantly higher than those in adjacent tissues.

Most of our experimental results are consistent with bioinformatics analysis and have shown that the expression levels of PD-L1, IL-10 and Met in ESCA tissues were higher than those in normal tissues. PD-L1 and IL-10 expression levels were positively correlated. Higher IL-10 expression levels are associated with advanced pathological staging of ESCA. However, some results are inconsistent with bioinformatics analysis. The survival analysis has shown that survival times of patients with higher IL10 expression levels were shorter than those with lower IL-10 expression levels-although the results were not statistically significant. In our study, we found that higher PD-L1 expression levels are associated with advanced pathological staging of ESCA. The result is different from TCGA. These different reasons might be best described as follows. First, the TCGA database generally collects tumor information from European and American research studies. 
A

Eca109 cells
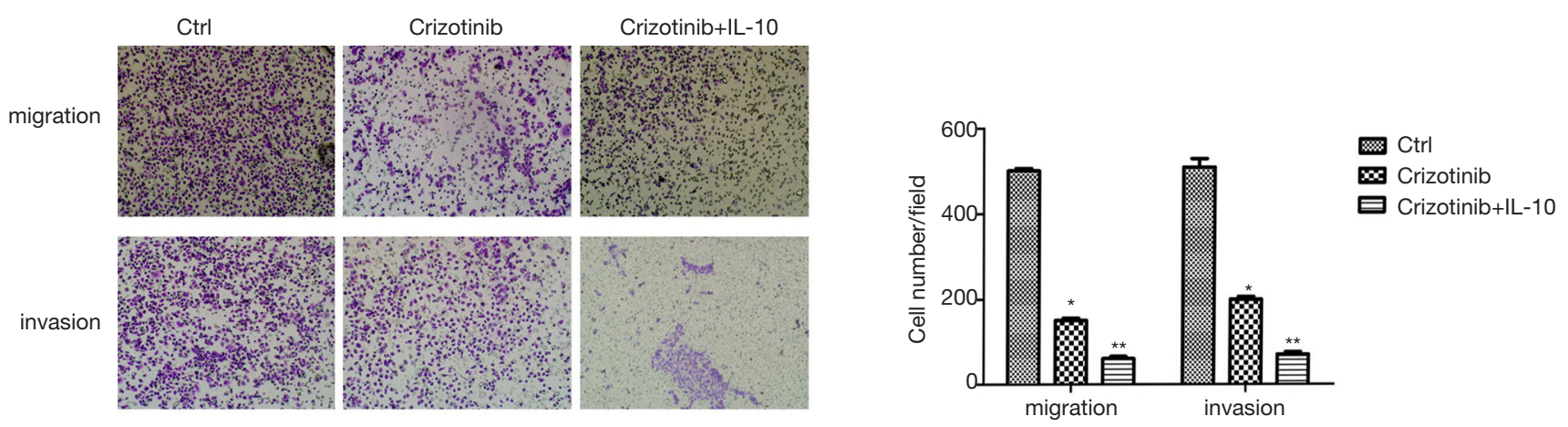

B

Ctrl

Crizotinib

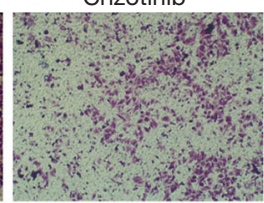

Crizotinib+IL-10

migration

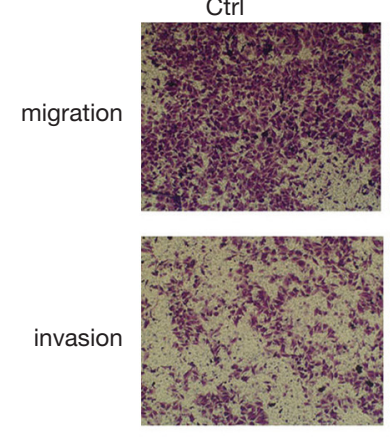

TE-1 cells
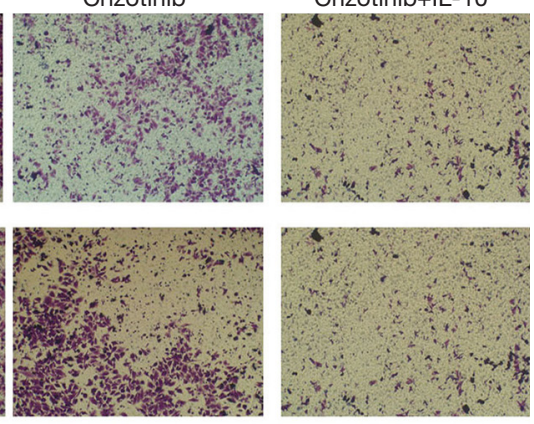

C
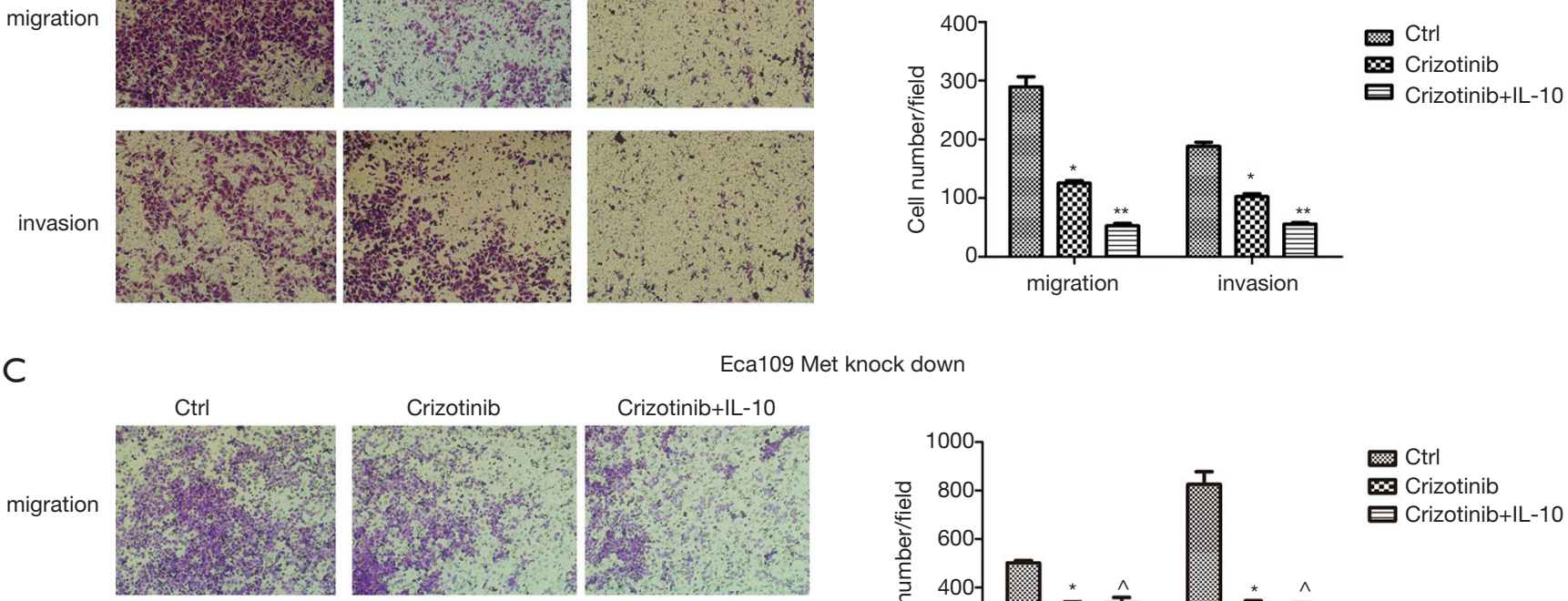

Crizotinib+IL-10
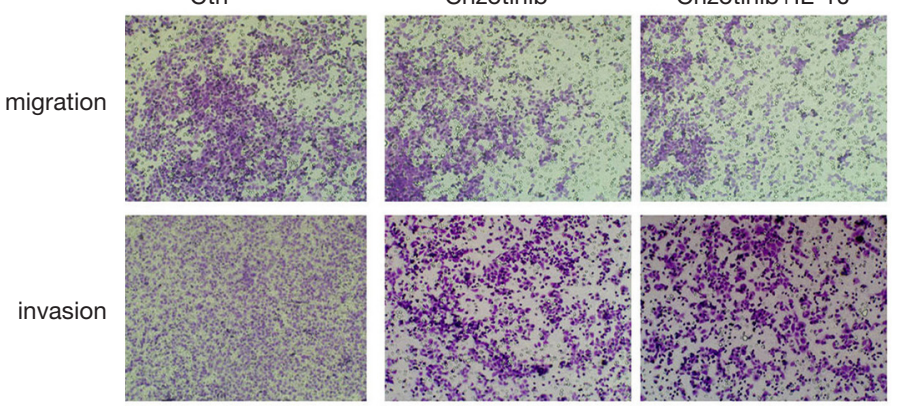

D
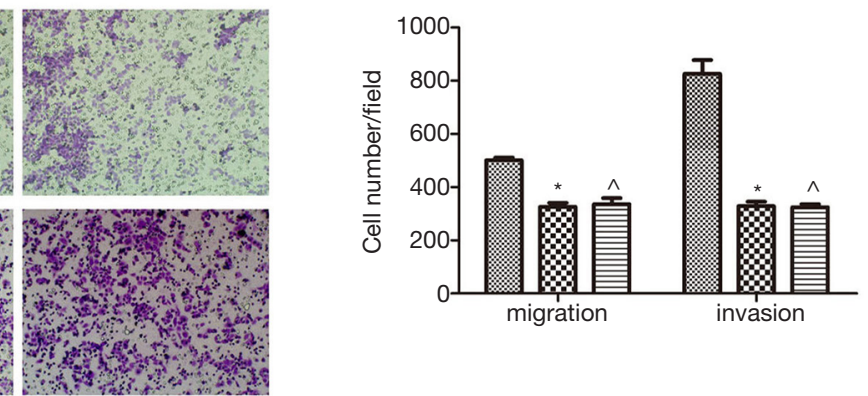

Ctrl

$\infty$ Crizotinib

Crizotinib+IL-10

TE-1 Met knock down

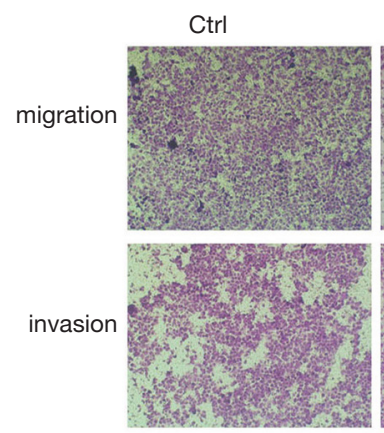

Crizotinib

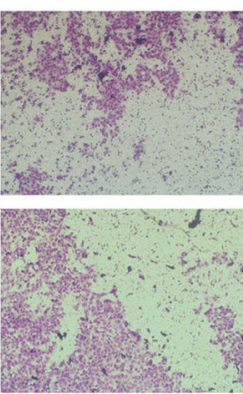

Crizotinib+IL-10
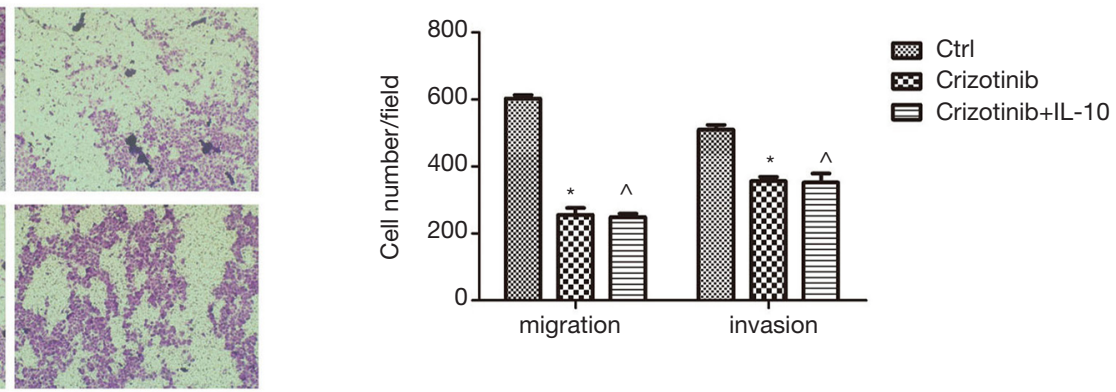

(C) Journal of Gastrointestinal Oncology. All rights reserved. 

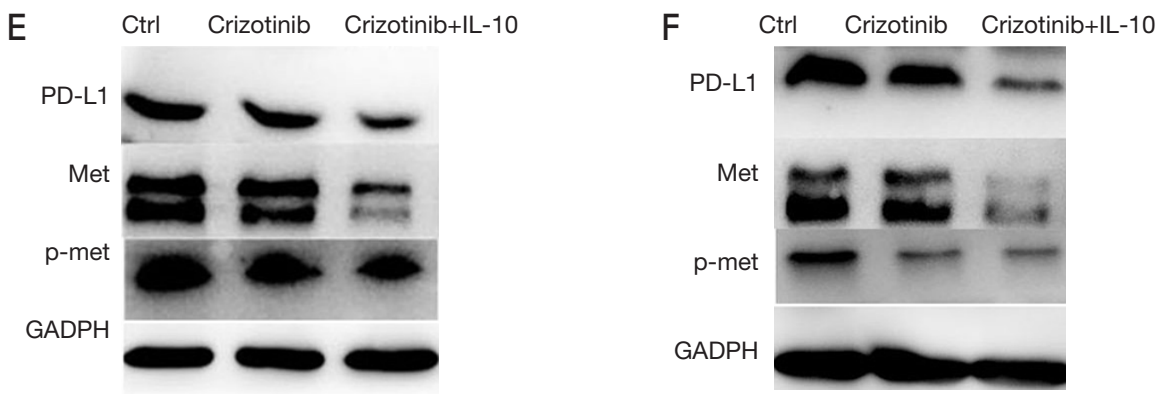

Figure 7 Effects of IL-10 and crizotinib on proliferation, migration invasion and of esophageal squamous cell lines. (A,B) Effects of crizotinib and combination of crizotinib and IL-10 on invasion and migration of esophageal squamous cell lines. (C,D) Effects of crizotinib and combination of crizotinib and IL-10 on invasion and migration of esophageal squamous cell lines after Met gene was knocked down in two cell lines. (E,F) Western blot experiments for the effects of crizotinib and combination of IL-10 and crizotinib on expression of PDL1, Met signal pathway in two cell lines. *, $\mathrm{P}<0.05$ compared with control group. ** $\mathrm{P}<0.05$ compared with crizotinib group. $\wedge$, $\mathrm{P} \geq 0.05$ compared with crizotinib group. Staining method: crystal violet staining. Magnification: $\times 200$.
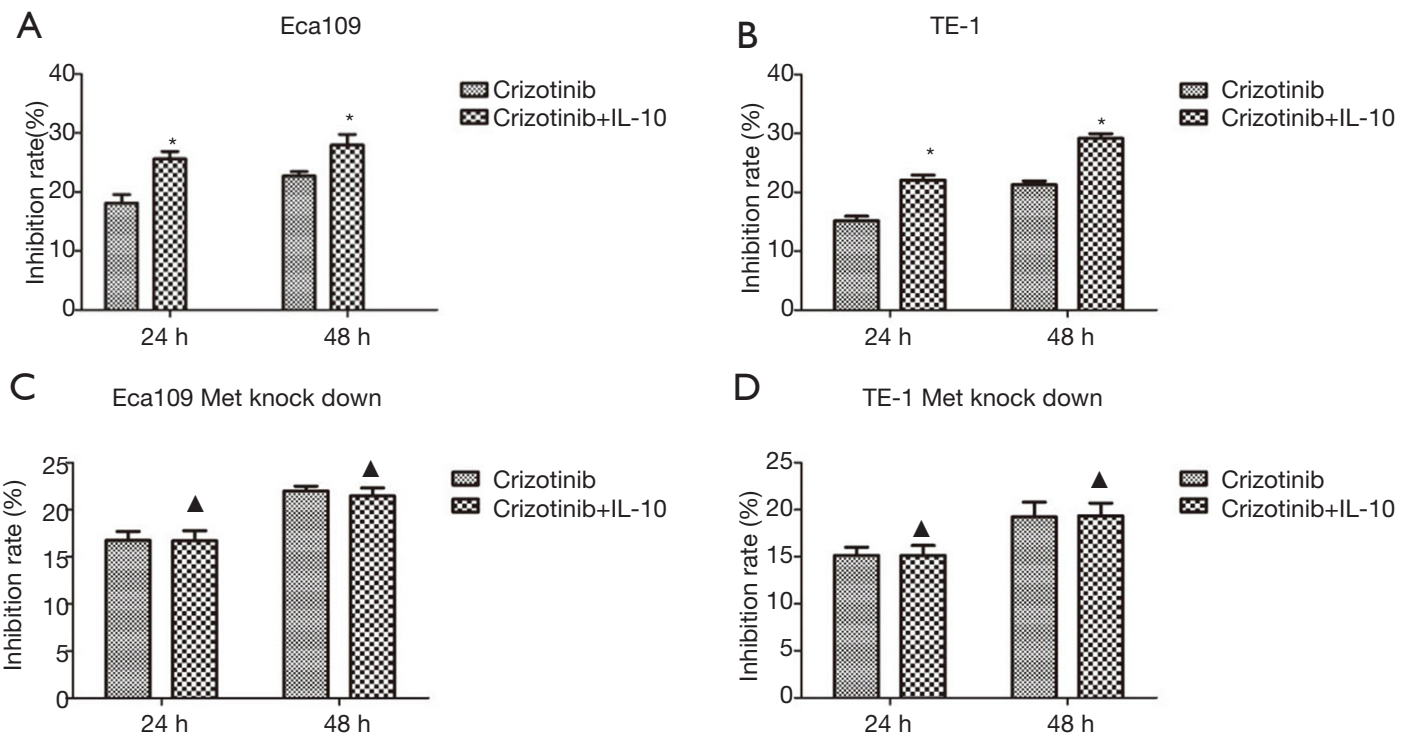

D TE-1 Met knock down

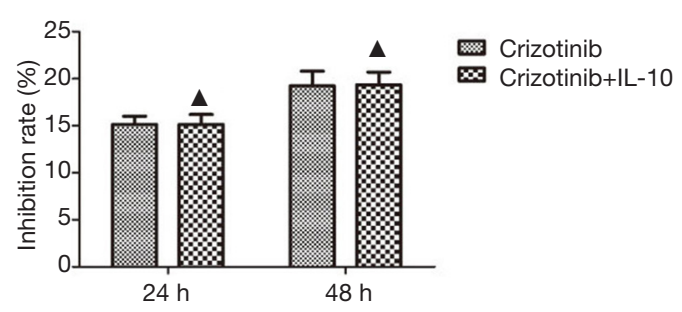

Figure 8 Effects of IL-10 and crizotinib on proliferation of esophageal squamous cell lines. (A,B) Effect of crizotinib combined with crizotinib and IL-10 on proliferation. (C,D) Effect of crizotinib combined with crizotinib and IL-10 on proliferation of Eca109 and TE-1 after Met gene was knocked out.*, $\mathrm{P}<0.05$ compared with crizotinib group; $\boldsymbol{\Delta}, \mathrm{P} \geq 0.05$ compared with crizotinib group.

Differences in expression levels of related factors were due to ethnic differences. Second, the TCGA database generally uses high-throughput sequencing to detect the expression at the gene level, which might not be different at the protein level. Third, due to the difference in sample numbers, data reported in the current study was different from the results of TCGA.

In tissue experiments, we found that the expression levels of PD-L1 and IL-10 were higher in cancer tissues than in adjacent tissues. There was also a positive correlation between the two factors. Similarly, in cellular experiments, we found that over-expression of PD-L1 can over-activate the Met signaling pathway, thereby up-regulating IL-10 expression. Thus, we infer that whether in cancer tissues or cells, over-expressed PD-L1 can activate the Met signaling pathway via positive feedback regulation, thereby upregulating IL-10 expression. Instead, we also found that IL10 down-regulates the expression of PD-L1, and anti-IL-10 
up-regulates the expression of PD-L1 through the Met signal pathway. Thus we infer that IL-10 can down-regulate PD-L1 expression by inhibiting the Met signaling pathway and the down-stream MAPK signaling pathway via negative feedback regulation (Figure 1B,C).

Some previously published studies suggest that IL10 is an immunological negative regulator and inhibits the function of T, B and dendritic cells and up-regulates PD-L1 expression (28-30,43). Recent data have shown that IL-10 release might be mediated following PD-L1 blockade, which sustains immunosuppression in ovarian cancer (29). HGF/MET signaling is essential for cancer survival (44) and important in the interaction between IL10 and PD-L1, which is involved in the immune response, including an increase in IL-10 (45-47) and PD-L1 which co-localizes with MET (48). Thus, in our results, IL-10 down-regulates PD-L1 expression, and does so probably by blocking the Met signaling pathway and the downstream MAPK signaling pathway. When the Met gene was knocked down, we observed no significant differences in the PDL1 expression levels in different intervention groups. The results demonstrated that the Met signaling pathway plays an important role between IL-10 and PD-L1. The role of IL-10 is complex and multifaceted. IL-10 promotes immune activation, increases tumor-specific immune surveillance and reduces the occurrence of pathogenic inflammatory reactions by activating $\mathrm{T}$ and $\mathrm{NK}$ cells.

Reportedly, IL-10 inhibits IC function and up-regulates PD-L1 expression $(28-30,43)$. However, our study suggests that IL-10 activates IC function, inhibits the Met signaling pathway, and down-regulates PD-L1 expression in ESCA cells. The results provide new mechanisms concerning the interaction between IL-10 and PD-L1, which might benefit clinical applications in the near future. Therefore, more in vivo and in vitro experiments are arranged to determine the involved modes and mechanisms of action. Our research of course, has still much follow-up work with a deeper exploration of the molecular mechanism of this phenomenon being required.

\section{Acknowledgments}

Funding: None.

\section{Footnote}

Conflicts of Interest: All authors have completed the ICMJE uniform disclosure form (available at http://dx.doi. org/10.21037/jgo.2020.01.06). The authors have no conflicts of interest to declare.

Ethical Statement: The authors are accountable for all aspects of the work in ensuring that questions related to the accuracy or integrity of any part of the work are appropriately investigated and resolved. The work was performed in accordance with the local Ethics Committee of the Third Affiliated Hospital of Soochow University (No. 20110140). Written informed consent was obtained from each patient, including signed consent for tissue analysis and consent to be recorded for potential medical research at the time of sample acquisition.

Open Access Statement: This is an Open Access article distributed in accordance with the Creative Commons Attribution-NonCommercial-NoDerivs 4.0 International License (CC BY-NC-ND 4.0), which permits the noncommercial replication and distribution of the article with the strict proviso that no changes or edits are made and the original work is properly cited (including links to both the formal publication through the relevant DOI and the license). See: https://creativecommons.org/licenses/by-nc$\mathrm{nd} / 4.0 /$.

\section{References}

1. Ferlay J, Soerjomataram I, Dikshit R, et al. Cancer incidence and mortality worldwide: sources, methods and major patterns in GLOBOCAN 2012. Int J Cancer 2015;136:E359-86.

2. Pennathur A, Gibson MK, Jobe BA, et al. Oesophageal carcinoma. Lancet 2013;381:400-12.

3. Kelsen DP, Ginsberg R, Pajak TF, et al. Chemotherapy followed by surgery compared with surgery alone for localized esophageal cancer. N Engl J Med 1998;339:1979-84.

4. van Hagen P, Hulshof MC, van Lanschot JJ, et al. Preoperative chemoradiotherapy for esophageal or junctional cancer. N Engl J Med 2012;366:2074-84.

5. Pardoll DM. The blockade of immune checkpoints in cancer immunotherapy. Nat Rev Cancer 2012;12:252-64.

6. Ribas A. Tumor immunotherapy directed at PD-1. N Engl J Med 2012;366:2517-9.

7. Blank C, Mackensen A. Contribution of the PD-L1/PD-1 pathway to T-cell exhaustion: an update on implications for chronic infections and tumor evasion. Cancer Immunol Immunother 2007;56:739-45. 
8. Ashraf N. Atezolizumab Treatment of Nonsquamous NSCLC. N Engl J Med 2018;379:1187-8.

9. Jung KH, LoRusso P, Burris H, et al. Phase I Study of the Indoleamine 2, 3-Dioxygenase 1 (IDO1) Inhibitor Navoximod (GDC-0919) Administered with PD-L1 Inhibitor (Atezolizumab) in Advanced Solid Tumors. Clin Cancer Res 2019;25:3220-8.

10. Meindl-Beinker NM, Betge J, Gutting T, et al. A multicenter open-label phase II trial to evaluate nivolumab and ipilimumab for 2nd line therapy in elderly patients with advanced esophageal squamous cell cancer (RAMONA). BMC Cancer 2019;19:231.

11. Hamilton $G$, Rath B. Immunotherapy for small cell lung cancer: mechanisms of resistance. Expert Opin Biol Ther 2019;19:423-32.

12. Maughan BL, Bailey E, Gill DM, et al. Incidence of Immune-Related Adverse Events with Program Death Receptor-1 - and Program Death Receptor-1 LigandDirected Therapies in Genitourinary Cancers. Front Oncol 2017;7:56.

13. Raju S, Joseph R, Sehgal S. Review of checkpoint immunotherapy for the management of non-small cell lung cancer. Immunotargets Ther 2018;7:63-75.

14. Chowdhury PS, Chamoto K, Honjo T. Combination therapy strategies for improving PD-1 blockade efficacy: a new era in cancer immunotherapy. J Intern Med 2018;283:110-20.

15. Esteva FJ, Hubbard-Lucey VM, Tang J, et al. Immunotherapy and targeted therapy combinations in metastatic breast cancer. Lancet Oncol 2019;20:e175-86.

16. Bylicki O, Barazzutti H, Paleiron N, et al. First-Line Treatment of Non-Small-Cell Lung Cancer (NSCLC) with Immune Checkpoint Inhibitors. BioDrugs 2019;33:159-71.

17. Cyprian FS, Akhtar S, Gatalica Z, et al. Targeted immunotherapy with a checkpoint inhibitor in combination with chemotherapy: A new clinical paradigm in the treatment of triple-negative breast cancer. Bosn J Basic Med Sci 2019;19:227-33.

18. Gajiwala S, Torgeson A, Garrido-Laguna I, et al. Combination immunotherapy and radiation therapy strategies for pancreatic cancer-targeting multiple steps in the cancer immunity cycle. J Gastrointest Oncol 2018;9:1014-26.

19. Wirsdörfer F, de Leve S, Jendrossek V. Combining Radiotherapy and Immunotherapy in Lung Cancer: Can We Expect Limitations Due to Altered Normal Tissue Toxicity? Int J Mol Sci 2018. doi: 10.3390/ijms20010024.
20. Hutmacher C, Gonzalo Nunez N, Liuzzi AR, et al. Targeted Delivery of IL2 to the Tumor Stroma Potentiates the Action of Immune Checkpoint Inhibitors by Preferential Activation of NK and CD8 (+) T Cells. Cancer Immunol Res 2019;7:572-83.

21. Li J, Xu J, Yan X, et al. Targeting Interleukin-6 (IL-6) Sensitizes Anti-PD-L1 Treatment in a Colorectal Cancer Preclinical Model. Med Sci Monit 2018;24:5501-8.

22. Menssen HD, Harnack U, Erben U, et al. Antibody-based delivery of tumor necrosis factor (L19-TNFalpha) and interleukin-2 (L19-IL2) to tumor-associated blood vessels has potent immunological and anticancer activity in the syngeneic J558L BALB/c myeloma model. J Cancer Res Clin Oncol 2018;144:499-507.

23. Ip WKE, Hoshi N, Shouval DS, et al. Anti-inflammatory effect of IL-10 mediated by metabolic reprogramming of macrophages. Science 2017;356:513-9.

24. Zielinski CE, Mele F, Aschenbrenner D, et al. Pathogeninduced human TH17 cells produce IFN-gamma or IL-10 and are regulated by IL-1beta. Nature 2012;484:514-8.

25. Chechlinska M, Kowalewska M, Nowak R. Systemic inflammation as a confounding factor in cancer biomarker discovery and validation. Nat Rev Cancer 2010;10:2-3.

26. Danilova AB, Danilov AO, Fakhrutdinova OL, et al. Laboratory evaluation of TGFbeta1, IL-10, VEGF levels in vivo and in vitro in patients with solid tumors. Vopr Onkol 2011;57:759-66.

27. Wang DZ, Zhang XH, Wu WX, et al. Exploration of the association of $\mathrm{H}$. pylori and EBV infection with cardiac and distal gastric adenocarcinoma among residents in Cixian County, a high-risk area of esophageal cancer in Hebei province. Zhonghua Zhong Liu Za Zhi 2012;34:446-9.

28. Brooks DG, Ha SJ, Elsaesser H, et al. IL-10 and PDL1 operate through distinct pathways to suppress T-cell activity during persistent viral infection. Proc Natl Acad Sci U S A 2008;105:20428-33.

29. Lamichhane P, Karyampudi L, Shreeder B, et al. IL-10 Release upon PD-1 Blockade Sustains Immunosuppression in Ovarian Cancer. Cancer Res 2017;77:6667-78.

30. Ruffner MA, Kim SH, Bianco NR, et al. B7-1/2, but not PD-L1/2 molecules, are required on IL-10-treated tolerogenic DC and DC-derived exosomes for in vivo function. Eur J Immunol 2009;39:3084-90.

31. Wang J, Rodriquez G, Norcross MA. Control of adaptive immune responses by Staphylococcus aureus through IL10, PD-L1, and TLR2. Sci Rep 2012;2:606.

32. Zhang J, Benedek G, Bodhankar S, et al. IL-10 producing 
B cells partially restore E2-mediated protection against EAE in PD-L1 deficient mice. J Neuroimmunol 2015;285:129-36.

33. Smyth EC, Sclafani F, Cunningham D. Emerging molecular targets in oncology: clinical potential of MET/ hepatocyte growth-factor inhibitors. Onco Targets Ther 2014;7:1001-14.

34. Zhang Y, Xia M, Jin K, et al. Function of the c-Met receptor tyrosine kinase in carcinogenesis and associated therapeutic opportunities. Mol Cancer 2018;17:45.

35. Kashyap MK, Abdel-Rahman O. Expression, regulation and targeting of receptor tyrosine kinases in esophageal squamous cell carcinoma. Mol Cancer 2018;17:54.

36. Yang Y, Fa X. Role of IL-10 gene polymorphisms on the susceptibility for esophageal cancer and its association with environmental factors. Int J Clin Exp Pathol 2015;8:9580-5.

37. Chen L, Deng H, Lu M, et al. B7-H1 expression associates with tumor invasion and predicts patient's survival in human esophageal cancer. Int J Clin Exp Pathol 2014;7:6015-23

38. Hino R, Kabashima K, Kato Y, et al. Tumor cell expression of programmed cell death-1 ligand 1 is a prognostic factor for malignant melanoma. Cancer 2010;116:1757-66.

39. Ito S, Okano S, Morita M, et al. Expression of PD-L1 and HLA Class I in Esophageal Squamous Cell Carcinoma: Prognostic Factors for Patient Outcome. Ann Surg Oncol 2016;23:508-15.

40. Imai D, Yoshizumi T, Okano S, et al. IFN-gamma Promotes Epithelial-Mesenchymal Transition and the Expression of PD-L1 in Pancreatic Cancer. J Surg Res 2019;240:115-23.

41. Schumacher K, Haensch W, Roefzaad C, et al. Prognostic significance of activated CD8 (+) T cell infiltrations within esophageal carcinomas. Cancer Res 2001;61:3932-6.

42. Tsutsumi S, Saeki H, Nakashima Y, et al. Programmed death-ligand 1 expression at tumor invasive front is associated with epithelial-mesenchymal transition and poor prognosis in esophageal squamous cell carcinoma. Cancer Sci 2017;108:1119-27.

43. Getts DR, Turley DM, Smith CE, et al. Tolerance induced by apoptotic antigen-coupled leukocytes is induced by PD-L1+ and IL-10-producing splenic macrophages and maintained by T regulatory cells. J Immunol 2011;187:2405-17.

44. Papaccio F, Della Corte CM, Viscardi G, et al. HGF/ MET and the Immune System: Relevance for Cancer Immunotherapy. Int J Mol Sci 2018. doi: 10.3390/ ijms19113595.

45. Okunishi K, Dohi M, Nakagome K, et al. A novel role of hepatocyte growth factor as an immune regulator through suppressing dendritic cell function. J Immunol 2005;175:4745-53.

46. Rutella S, Bonanno G, Procoli A, et al. Hepatocyte growth factor favors monocyte differentiation into regulatory interleukin (IL)-10++IL-12low/neg accessory cells with dendritic-cell features. Blood 2006;108:218-27.

47. Shintani Y, Aoki H, Nishihara M, et al. Hepatocyte growth factor promotes an anti-inflammatory cytokine profile in human abdominal aortic aneurysm tissue. Atherosclerosis 2011;216:307-12.

48. Balan M, Mier y Teran E, Waaga-Gasser AM, et al. Novel roles of c-Met in the survival of renal cancer cells through the regulation of HO-1 and PD-L1 expression. J Biol Chem 2015;290:8110-20.
Cite this article as: Qian Q, Wu C, Chen J, Wang W. Relationship between IL-10 and PD-L1 in esophageal carcinoma tissues and IL-10 down-regulates PD-L1 expression via Met signaling pathway. J Gastrointest Oncol 2020;11(2):337355. doi: 10.21037/jgo.2020.01.06 


\section{Immunochemistry}

The paraffin-embedded tumor samples were sectioned to $5 \mu \mathrm{m}$ slices. The tissue section slides were deparaffinized and then rehydrated. For PD-L1 and IL-10 immunochemical staining, antigen retrieval was achieved in boiled EDTA for 30 min. The tissue section slices were incubated with peroxidase blocking reagent $\left(3 \% \mathrm{H}_{2} \mathrm{O}_{2}\right.$ solution) for $30 \mathrm{~min}$. The slices were incubated with the primary PD-L1 antibody (Novus ittleton, Colorado, USA) and IL-10 antibody (Novus, Littleton, Colorado, USA) in a humidified chamber overnight at $4{ }^{\circ} \mathrm{C}$. After being washed three times in PBS, the slices were then incubated with anti-mouse/rabbit secondary antibody (Maixin, Fuzhou, Fujian, China) in a dedicated chamber for 30 min. We than stained the slices with hematoxylin solution.

The results were evaluated by two independent pathologists at the Third Affiliated Hospital of Suzhou University. The intensity was classified as follows: 0 , negative staining; 1 , weak staining; 2 , moderate staining; 3 , strong staining. The rate of positive cells was recorded: $1: 0-25 \% ; 2: 26-50 \% ; 3: 51-75 \%$; and 4 : greater than $75 \%$. A final score was achieved by multiplying the staining intensity $(0,1,2$, and 3$)$ and the rate of positive cells observed (1, 2, 3 and 4). For data analysis, scores of less than 8 were defined as low expression and scores of 8 or more, as high expression.

\section{Quantitative RT-PCR}

The tissue pieces were first cut into small pieces, and then a homogenizer was used to extract total RNA using the Trizol method. We compared the differences in IL-10, PD-L1, and Met mRNA expression among cancerous, adjacent, and normal tissues. The primers (Sangon, Shanghai, China) were listed in the supplementary material. A Takara (Takara, Tokyo, Japan) RT-PCR Kit were used for this assay.

\section{Cell culture}

The esophageal squamous cell-lines Eca109 and TE-1 were from the obtained from the Cell Research Center, Third Affiliated Hospital of Soochow University for in vitro studies. Eca109 cells were cultured in RPMI 1640 medium (Hyclone, Logan City, Utah, USA) and supplemented with 10\% fetal calf serum (FCS; Gibco, Thermo-Fisher Scientific, Grand Island, NY, USA), 100 units/mL penicillin, and $100 \mathrm{ug} / \mathrm{mL}$ streptomycin. TE-1 cells were cultured in DMEM medium (Hyclone, Logan City, Utah, USA), which was supplemented with $10 \%$ FCS, 100 units $/ \mathrm{mL}$ penicillin, and $100 \mathrm{ug} / \mathrm{mL}$ streptomycin. Cells were incubated at $37{ }^{\circ} \mathrm{C}$ in a humidified atmosphere containing $5 \% \mathrm{CO}_{2}$ in air. Cells were used in the experiments when found to be in the logarithmic growth phase.

\section{SiRNA construction of esophageal squamous cell-lines}

One day before transfection, cells in the logarithmic growth phase were removed and seeded on a six-well plate at a density of $10^{4}-10^{5}$ per plate. We used customized siRNA (Gene Pharma Company, Shanghai, China) and configured an X-treme GENE siRNA Transfection Reagent—siRNA plasmid mixture, which was then added to the cells to be transfected. After 36 hours, the transfection effect was detected by PCR. If the transfection efficiency reached above 60 percent, then the cells could be used for the next experiment.

\section{CCK8}

One day before drug treatment, cells in the logarithmic growth phase were removed and seeded into a 96-well plate at a density of $2 \times 10^{3}$ cells per well $(100 \mathrm{uL} /$ well). Next, we used CCK8 (Dojindo, Tokyo, Japan) to detect the proliferation of cells after treatment with Crizotinib (Cell Signaling Technology, Danvers, MA, USA) and Crizotinib combined with IL-10. Lastly, we added Crizotinib or Crizotinib combined with IL-10 to the constructed siMet Eca109 and siMet TE-1. The proliferation of cells was detected by CCK8 assay. The inhibition rate is calculated by the following formula: inhibition rate $=[$ (control well-experimental well)/(control well-blank well)] $\times 100 \%$. 


\section{Western blot}

Total cellular protein was extracted by a protein extraction agent (KeyGen Biotech, Nanjing, Jiangsu, China) after 48 hours of treatment with different concentrations of IL-10, anti-IL-10 antibody. Protein concentrations were determined by a bicinchoninic acid assay (BCA assay, Pierce, Thermo-Fisher Scientific, Waltham, MA, USA). Aliquots of protein lysates were separated on sodium dodecyl sulfate-polyacrylamide gels (SDS-PAGE) and transferred onto a PVDF membrane, which was blocked with $3 \%$ BSA in TBST. The membrane was then hybridized with primary antibodies. Then, with the corresponding secondary antibodies conjugated with horseradish peroxidase, the specific protein bands on the membrane were detected by a chemiluminescence assay (EMD Millipore, Temecula, CA, USA). GADPH was used as a loading control. All primary and secondary antibodies used in the Western blotting assay were purchased from Cell Signaling Technology, Abcam or the Novus Company. PD-L1 expression levels, Met signaling pathway-related molecules (Met and phospho-Met) and their downstream MAPK signaling pathway-related molecules (akt, phospho-akt, Mek, phospho-Mek, Erk, phospho-Erk) were analyzed. In addition, we performed Western blotting on esophageal cancer cells that overexpressed or were knocked down in the expression of the PD-L1 gene to detect differences in IL-10 expression levels. Subsequently, crizotinib or crizotinib combined with IL-10 were added to the cells. Met signaling pathway-related molecules (Met and phospho-Met) were analyzed.

In our tissue experiments, the tissue specimens were first cut into small sections, and then a homogenizer was used to extract tissue protein using the protein extraction reagent. We compared the differences in IL-10, PD-L1, and Met expression among cancerous, adjacent and normal tissues.

Table S1 Score for cell intensity or the rate of positive cells

\begin{tabular}{lll}
\hline Score & Degree of staining & The rate of positive cells \\
\hline 1 & Negative staining & $0-25 \%$ \\
2 & Moderate staining & $26-50 \%$ \\
3 & Strong staining & $51-75 \%$ \\
4 & - & $>75 \%$ \\
\hline
\end{tabular}

Table S2 Primers for RT-PCR

\begin{tabular}{ll}
\hline Gene & Sequence \\
\hline IL-10 & Forward 5'-CTT CGA GAT CTC CGA GAT GCC TTC-3' \\
& Reverse 5'-ATT CTT CAC CTG CTC CAC GGC CTT-3' \\
fet & Rerward 5'-GGT TCA CTG CAT ATT CTC CCC-3' \\
& Reverse 5'-ACC ATC TTT CGT TTC CTT TAG CC-3' \\
PD-L1 & Rerward 5'-ACT GGC ATT TGC TGA ACG-3' \\
& Foverse 5'-TCC TCC ATT TCC CAA TAG AC-3' \\
GADPH & Reverse 5'-CAC CCT GTT GCT GTA GCC AAA-3' \\
\end{tabular}

Table S3 siRNA of Met and PD-L1

\begin{tabular}{ll}
\hline siRNA & Sequence \\
\hline siMet & Sense: 5'-CTC ATT TGG ATA GGC TTG TAA-3' \\
& Anti-sense: 5'-TTA CAA GCC TAT CCA AAT GAG-3' \\
siPD-L1 & Sense: 5'-CAG AAA GAU GAG GAU AUUU-3' \\
& Anti-sense: 5'-AAA UAU CCU CAU CUU UCUG-3' \\
\hline
\end{tabular}

